\title{
Anti-norms on Finite von Neumann Algebras
}

\author{
by \\ Jean-Christophe Bourin and Fumio HiaI
}

\begin{abstract}
As the reversed version of usual symmetric norms, we introduce the notion of symmetric anti-norms $\|\cdot\|$ ! defined on the positive operators affiliated with a finite von Neumann algebra with a finite normal trace. Related to symmetric anti-norms, we develop majorization theory and superadditivity inequalities of the form $\|\psi(A+B)\| ! \geq\|\psi(A)\| !+\|\psi(B)\|$ ! for a wide class of functions $\psi$.
\end{abstract}

2010 Mathematics Subject Classification: 47A30, 46L52, 46L51.

Keywords: finite von Neumann algebra, symmetric norm, symmetric anti-norm, $\tau$-measurable operator, majorization, unitary orbit, spectral dominance, Fuglede-Kadison determinant.

\section{$\S 1$. Introduction}

In functional analysis, symmetrically normed Banach function spaces are classical objects, as also are their non-commutative generalizations in the setting of $\tau$-measurable operators affiliated with a von Neumann algebra with a faithful normal finite trace $\tau$. Symmetric norms are homogeneous convex functionals completely determined by their values on the positive cone of the function space or the operator algebra. This point of view motivates the study of concave, homogeneous functionals on positive operators. It is our concern in this article.

A part of our work could fit in a very general setting, for instance, in the $C^{*}$ algebra framework. However, we confine ourselves to finite von Neumann algebras for two reasons. First, dealing with a von Neumann algebra equipped with a normal finite trace allows us to consider unbounded operators, and hence to develop a theory parallel to most of the usual non-commutative Banach function spaces.

Communicated by N. Ozawa. Received September 16, 2014. Revised December 10, 2014.

J.-C. Bourin: Laboratoire de mathématiques, Université de Franche Comté,

25030 Besançon, France;

e-mail: jcbourin@univ-fcomte.fr

F. Hiai: Tohoku University (Emeritus), Hakusan 3-8-16-303, Abiko 270-1154, Japan; e-mail: hiai.fumio@gmail.com 
Secondly, the finiteness assumption allows us to consider many functionals which would not make sense in the non-finite case, such as the Fuglede-Kadison determinant. The assumption is also essential for some technical reasons (for instance, a unitary operator can be taken as a phase of the polar decomposition). Moreover, this setup of a finite von Neumann algebra naturally extends the matrix approach in our previous work.

We call our functionals, defined on the positive part $\mathcal{N}^{+}$of a finite von Neumann algebra $\mathcal{N}$, symmetric anti-norms on $\mathcal{N}^{+}$, as the triangle inequality for norms is then reversed. Section 2 gives the precise definition and exhibits an important family of such anti-norms which are derived from symmetric norms. For the convenience of the reader and to fix terminology which has some variants in the literature, our discussion also covers basic facts on symmetric norms. Our approach to symmetric norms may be of independent interest. Section 4 is devoted to the (non-obvious) extension of these anti-norms to the whole set of densely-defined positive operators affiliated with $\mathcal{N}$. Here we consider the more classical case of symmetric norms as well. Section 5 presents a superadditivity inequality for convex functions which is a far-reaching extension of a classical trace inequality of Rotfel'd. Several norm inequalities follow from this anti-norm inequality. In Sections 6 and 7 , we focus on a special class of symmetric norms and anti-norms which corresponds, in the commutative case, to the class of rearrangement invariant function spaces. The theory is then related to majorization relations.

Most of the results, norm and anti-norm inequalities given in Sections 4-7, are based on operator inequalities via unitary orbits. These essential operator inequalities are established in Section 3. The idea of the proofs consists in combining a unitary orbit technique for spectral dominance in a finite factor with the disintegration of $\mathcal{N}$ into its factorial components. These results nicely extend the scope of some well-known matrix inequalities to the general finite von Neumann algebra setting.

\section{§2. Symmetric norms and symmetric anti-norms}

Let $\mathcal{N}$ be a finite von Neumann algebra acting on a separable Hilbert space $\mathcal{H}$ with a faithful normal finite trace $\tau$, and $\mathcal{N}^{+}$the set of positive operators in $\mathcal{N}$. Let $\overline{\mathcal{N}}$ denote the set of $\tau$-measurable operators affiliated with $\mathcal{N}$ (see [13] for details), and $\overline{\mathcal{N}}^{+}$the positive cone of $\overline{\mathcal{N}}$. Since $\tau$ is finite, $\overline{\mathcal{N}}$ is the set of all densely-defined closed operators affiliated with $\mathcal{N}$.

In this article, a symmetric norm $\|\cdot\|$ on $\mathcal{N}$ means a norm satisfying $\|U X V\|=$ $\|X\|$ for all $X \in \mathcal{N}$ and all unitaries $U, V \in \mathcal{N}$. The monotonicity of such a norm in the next lemma is a well-known simple fact [14, Lemma 3.2, Corollary 3.3]. We give an alternative proof. The letter $I$ stands for the identity (of any algebra). 
Lemma 2.1. Any symmetric norm $\|\cdot\|$ on $\mathcal{N}$ is monotone, i.e., $\|A\| \leq\|B\|$ if $A, B \in \mathcal{N}^{+}$and $A \leq B$.

Proof. Let $T \in \mathcal{N}$ be a contraction. Note that $|T|=\left(V_{1}+V_{2}\right) / 2$ where $V_{1}:=$ $|T|+i \sqrt{I-|T|^{2}}$ and $V_{2}:=|T|-i \sqrt{I-|T|^{2}}$ are unitaries. As $T=U|T|$ for some unitary $U \in \mathcal{N}$ ( since $\mathcal{N}$ is a finite von Neumann algebra), we have $T=\left(U_{1}+U_{2}\right) / 2$ with two unitaries $U_{1}, U_{2} \in \mathcal{N}$. Therefore $\|T X T\| \leq\|X\|$ for all $X \in \mathcal{N}$. Now, assume that $0 \leq A \leq B$ in $\mathcal{N}$. Then there exists a contraction $C \in \mathcal{N}^{+}$such that $A=B^{1 / 2} C B^{1 / 2}=V C^{1 / 2} B C^{1 / 2} V^{*}$ for some unitary $V \in \mathcal{N}$. Hence $\|A\|=$ $\left\|C^{1 / 2} B C^{1 / 2}\right\| \leq\|B\|$.

Consequently, any symmetric norm on $\mathcal{N}$ is continuous with respect to the operator norm $\|\cdot\|_{\infty}$. In fact, since $|X| \leq\|X\|_{\infty} I$, we have

$$
\|X\| \leq\|X\|_{\infty}\|I\|, \quad X \in \mathcal{N}
$$

A symmetric norm $\|\cdot\|$ on $\mathcal{N}$ depends only on its values on positive operators via the polar decomposition, and its restriction to $\mathcal{N}^{+}$satisfies

(1) $\|\alpha A\|=\alpha\|A\|$ for all $A \in \mathcal{N}^{+}$and all scalars $\alpha \geq 0$,

(2) $\|A\|=\left\|U A U^{*}\right\|$ for all $A \in \mathcal{N}^{+}$and all unitaries $U \in \mathcal{N}$,

(3) $\|A\| \leq\|A+B\| \leq\|A\|+\|B\|$ for all $A, B \in \mathcal{N}^{+}$.

The first inequality in (3) follows from Lemma 2.1. Conversely, if $\|\cdot\|$ is a nonnegative functional on $\mathcal{N}^{+}$satisfying (1)-(3), then $\|X\|:=\||X|\|$ for $X \in \mathcal{N}$ becomes a symmetric norm (more precisely, seminorm) on $\mathcal{N}$, as immediately shown by a triangle inequality in [1] or by Proposition 3.4 below.

We introduce the notion of symmetric anti-norms on the positive cone $\mathcal{N}^{+}$, by replacing the convexity/subadditivity of symmetric norms with concavity/superadditivity.

Definition 2.2. A symmetric anti-norm $\|\cdot\|$ ! on $\mathcal{N}^{+}$is a functional taking values in $[0, \infty)$ satisfying the following properties:

(1)! $\|\alpha A\| !=\alpha\|A\|$ ! for all $A \in \mathcal{N}^{+}$and all scalars $\alpha \geq 0$,

(2)! $\|A\|_{!}=\left\|U A U^{*}\right\|$ ! for all $A \in \mathcal{N}^{+}$and all unitaries $U \in \mathcal{N}$,

$(3) !\|A+B\|_{!} \geq\|A\|_{!}+\|B\|_{!}$for all $A, B \in \mathcal{N}^{+}$.

$(4) !\|A+\varepsilon I\| ! \searrow\|A\|$ ! as $\varepsilon \searrow 0$ for all $A \in \mathcal{N}^{+}$.

This definition was first introduced in $[6,7]$ for the matrix algebra $\mathbb{M}_{n}$. In the matrix case, (4)! is equivalent to the usual continuity with respect to the operator norm. 
Typical examples of symmetric anti-norms are $A \mapsto\left\{\tau\left(A^{q}\right)\right\}^{1 / q}, 0<q \leq 1$, and $A \mapsto\left\{\tau\left(A^{-1 / p}\right)\right\}^{-1 / p}, 0<p<\infty$. The latter is first defined on the invertible part of $\mathcal{N}^{+}$, and understood for non-invertible operators as

$$
\left\{\tau\left(A^{-p}\right)\right\}^{-1 / p}:=\lim _{\varepsilon \searrow 0}\left\{\tau\left((A+\varepsilon I)^{-p}\right)\right\}^{-1 / p},
$$

where the finiteness assumption $\tau(I)<\infty$ is essential to have non-trivial functionals on $\mathcal{N}^{+}$. These Schatten-like functionals with negative exponents are a special case of a more general family.

Definition 2.3. Fix a symmetric norm $\|\cdot\|$ on $\mathcal{N}$ and $p>0$. For each $A \in \mathcal{N}^{+}$, since $\left\|(A+\varepsilon I)^{-p}\right\|^{-1 / p}$ decreases as $\varepsilon \searrow 0$ by Lemma 2.1, we can define

$$
\|A\|_{!}:=\lim _{\varepsilon \searrow 0}\left\|(A+\varepsilon I)^{-p}\right\|^{-1 / p} .
$$

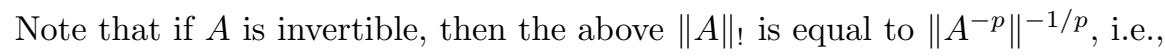

$$
\left\|A^{-p}\right\|^{-1 / p}=\lim _{\varepsilon \searrow 0}\left\|(A+\varepsilon I)^{-p}\right\|^{-1 / p} .
$$

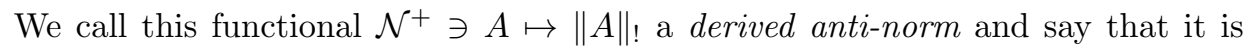
derived from $\|\cdot\|$ and $p$.

A derived anti-norm is indeed a symmetric anti-norm as claimed in the next statement.

Theorem 2.4. The above functional $\|\cdot\|$ ! derived from a symmetric norm $\|\cdot\|$ on $\mathcal{N}$ and a $p>0$ satisfies

$$
\|A+B\|_{!} \geq\|A\|_{!}+\|B\|_{!}
$$

for every $A, B \in \mathcal{N}^{+}$. Hence $\|\cdot\|$ ! is a symmetric anti-norm on $\mathcal{N}^{+}$.

To prove this result, we begin with an operator arithmetic-geometric mean inequality.

Lemma 2.5. Let $A, B \in \mathcal{N}^{+}$. Then there exists a unitary $V \in \mathcal{N}$ such that

$$
|B A| \leq \frac{A^{2}+V B^{2} V^{*}}{2} .
$$

Proof. Consider

$$
\left[\begin{array}{cc}
A^{2} & A B \\
B A & B^{2}
\end{array}\right]
$$


which is a positive operator on $\mathcal{H} \oplus \mathcal{H}$. Thus, for any $V \in \mathcal{N}$ so is

$$
\left[\begin{array}{ll}
I & -V
\end{array}\right]\left[\begin{array}{cc}
A^{2} & A B \\
B A & B^{2}
\end{array}\right]\left[\begin{array}{c}
I \\
-V^{*}
\end{array}\right] .
$$

Letting $V^{*}$ be the unitary factor in the polar decomposition $B A=V^{*}|B A|$ yields the desired inequality.

Combining Lemmas 2.1 and 2.5 yields $\|A B\| \leq\left(s\left\|A^{2}\right\|+s^{-1}\left\|B^{2}\right\|\right) / 2$ for all symmetric norms, $A, B \in \mathcal{N}^{+}$, and $s>0$. Thus, minimizing over $s$ and taking $A=\left|X^{*}\right|, B=\left|Y^{*}\right|$ for any operators $X, Y \in \mathcal{N}$, we obtain the Cauchy-Schwarz inequality for symmetric norms.

Corollary 2.6. Let $X, Y \in \mathcal{N}$. Then for any symmetric norm on $\mathcal{N}$,

$$
\left\|X^{*} Y\right\| \leq\left\|X^{*} X\right\|^{1 / 2}\left\|Y^{*} Y\right\|^{1 / 2} .
$$

As a byproduct of this inequality we get from $\|\cdot\|$ another symmetric norm.

Corollary 2.7. If $\|\cdot\|$ is a symmetric norm on $\mathcal{N}$, then so is $X \mapsto\left\|X^{*} X\right\|^{1 / 2}$.

For symmetric anti-norms, the following proposition is known in the matrix case $[6]$.

Lemma 2.8. If $\|\cdot\|$ ! is a symmetric anti-norm on $\mathcal{N}^{+}$and $0<q<1$, then $A \mapsto\left\|A^{q}\right\|_{!}^{1 / q}$ is also a symmetric anti-norm on $\mathcal{N}^{+}$.

Proof. The same proof as in the matrix case [6] shows that this is a homogeneous, unitarily invariant and concave functional. The continuity property (4)!, i.e., $\left\|A^{q}\right\|_{!}^{1 / q}=\lim _{\varepsilon \searrow 0}\left\|(A+\varepsilon I)^{q}\right\|_{!}^{1 / q}$, is obvious from the monotonicity of $\|\cdot\|$ ! since $A^{q} \leq(A+\varepsilon I)^{q} \leq A^{q}+\varepsilon^{q} I$.

We are now in a position to prove the theorem.

Proof of Theorem 2.4. Let $\|\cdot\|$ be a symmetric norm on $\mathcal{N}$. Let $A, B \in \mathcal{N}^{+}$be invertible and assume that $\left\|A^{-1}\right\|=\left\|B^{-1}\right\|=1$. As $t \mapsto t^{-1}$ is operator convex on $(0, \infty)$, we have, for $0<s<1$,

$$
\left\|(s A+(1-s) B)^{-1}\right\| \leq\left\|s A^{-1}+(1-s) B^{-1}\right\| \leq s+(1-s)=1
$$

and so

$$
\left\|(s A+(1-s) B)^{-1}\right\|^{-1} \geq 1 .
$$

For general invertible $S, T \in \mathcal{N}^{+}$, taking in this estimate $A=\left\|S^{-1}\right\| S, B=$ $\left\|T^{-1}\right\| T$, and $s=\left\|S^{-1}\right\|^{-1} /\left(\left\|S^{-1}\right\|^{-1}+\left\|T^{-1}\right\|^{-1}\right)$ yields

$$
\left\|(S+T)^{-1}\right\|^{-1} \geq\left\|S^{-1}\right\|^{-1}+\left\|T^{-1}\right\|^{-1} .
$$


Therefore, $A \mapsto\|A\|_{!}:=\left\|A^{-1}\right\|^{-1}$ is a homogeneous and concave/superadditive functional on the invertible part of $\mathcal{N}^{+}$. It can be extended with the same properties to the whole of $\mathcal{N}^{+}$by the limit formula $\|A\|_{!}:=\lim _{\varepsilon \searrow 0}\|A+\varepsilon I\|_{\text {!. Hence, }}$ this functional derived from a symmetric norm $\|\cdot\|$ and $p=1$ is a symmetric anti-norm on $\mathcal{N}^{+}$.

Next we consider a functional derived from a symmetric norm $\|\cdot\|$ and an arbitrary $p>0$. We have $p=2^{n} q$ where $n$ is a positive integer and $0<q<1$. By Corollary 2.7 applied $n$ times and the first step of the proof,

$$
\|A\|_{!}^{(n)}:=\lim _{\varepsilon \searrow 0}\left\|(A+\varepsilon I)^{-2^{n}}\right\|^{-1 / 2^{n}}
$$

is a symmetric anti-norm on $\mathcal{N}^{+}$. Applying Lemma 2.8 shows that $A \mapsto$ $\left(\left\|A^{q}\right\|_{!}^{(n)}\right)^{1 / q}$ is a symmetric anti-norm too, which is readily verified to be the functional derived from $\|\cdot\|$ and $p$.

\section{$\S 3$. Inequalities via unitary orbits}

This section is the main technical part of this article. The next theorems give superadditive or subadditive operator inequalities via unitary orbits for convex or concave functions, which will be of essential use in Sections 4 and 5 .

Theorem 3.1. Let $g$ be a non-negative convex function on $[0, \infty)$ with $g(0)=0$. Assume that either $\mathcal{N}$ is a factor and $A, B \in \overline{\mathcal{N}}^{+}$, or $A, B \in \mathcal{N}^{+}$without the factoriality assumption on $\mathcal{N}$. Then, for every $\varepsilon>0$, there exist unitaries $U, V \in \mathcal{N}$ such that

$$
g(A+B)+\varepsilon I \geq U g(A) U^{*}+V g(B) V^{*} .
$$

Theorem 3.2. Let $f$ be a non-negative concave function on $[0, \infty)$. Assume that either $\mathcal{N}$ is a factor and $A, B \in \overline{\mathcal{N}}^{+}$, or $A, B \in \mathcal{N}^{+}$without the factoriality of $\mathcal{N}$. Then, for every $\varepsilon>0$, there exist unitaries $U, V \in \mathcal{N}$ such that

$$
f(A+B) \leq U f(A) U^{*}+V f(B) V^{*}+\varepsilon I .
$$

Before proving the theorems we recall the notion of the spectral scale [26]. The spectral scale of $A \in \overline{\mathcal{N}}^{+}$is defined as

$$
\lambda_{t}(A):=\inf \left\{s \in \mathbb{R}: \tau\left(\mathbf{1}_{(s, \infty)}(A)\right) \leq t\right\}, \quad t \in(0, \tau(I)),
$$

where $\mathbf{1}_{(s, \infty)}(A)$ is the spectral projection of $A$ corresponding to $(s, \infty)$. We write $\lambda(A)$ for the function $t \mapsto \lambda_{t}(A)$ on $(0, \tau(I))$, which is non-increasing and right-continuous. Furthermore, we write $\lambda_{0}(A)$ and $\lambda_{\tau(I)}(A)$ for $\lim _{t \searrow_{0}} \lambda_{t}(A)$ and $\lim _{t \nearrow \tau(I)} \lambda_{t}(A)$, respectively, which are the maximal and minimal spectra 
of $A$ (when $A$ is bounded). The generalized s-numbers [13] of $X \in \overline{\mathcal{N}}$ are $\mu_{t}(X):=\lambda_{t}(|X|), t \in(0, \tau(I))$.

To prove the theorems we will use the following lemma. The lemma is rather well-known but we give the proof for the convenience of the reader.

Lemma 3.3. Let $\mathcal{N}$ be a finite factor and $A, B \in \overline{\mathcal{N}}^{+}$. If $B$ spectrally dominates $A$, i.e., $\lambda_{t}(A) \leq \lambda_{t}(B)$ for all $t \in(0, \tau(I))$, then for every $\varepsilon>0$ there exists a unitary $U \in \mathcal{N}$ such that $U A U^{*} \leq B+\varepsilon I$.

Proof. Since the matrix case is obvious without $\varepsilon I$ on the right-hand side, we may assume that $\mathcal{N}$ is a type $\mathrm{II}_{1}$ factor with the normalized trace $\tau$. Choose an increasing family $\left\{F_{t}\right\}_{0 \leq t \leq 1}$ of projections in $\mathcal{N}$ such that $\tau\left(F_{t}\right)=t$ for all $t \in[0,1]$.

Define

$$
\tilde{A}:=\int_{0}^{1} \lambda_{t}(A) d F_{t}, \quad \tilde{B}:=\int_{0}^{1} \lambda_{t}(B) d F_{t} .
$$

Then $\lambda(\tilde{A})=\lambda(A), \lambda(\tilde{B})=\lambda(B)$ and $\tilde{A} \leq \tilde{B}$. Hence the assertion follows since $\left\|A-V \tilde{A} V^{*}\right\|_{\infty}<\varepsilon / 2$ and $\left\|B-W \tilde{B} W^{*}\right\|_{\infty}<\varepsilon / 2$ for some unitaries $V, W \in \mathcal{N}$ by [18, Lemma 4.1].

We now turn to the proofs of the theorems, which are based on the spectral dominance theorem [9] and the central direct decomposition.

Proof of Theorem 3.1. First assume that $\mathcal{N}$ is a finite factor. Then the matrix case is $\left[2\right.$, Theorem 2.1] (without $\varepsilon I$ on the left-hand side). The proof in the type $\mathrm{II}_{1}$ factor case is similar based on [9]. For any contraction $Z \in \mathcal{N}$ and any $T \in \overline{\mathcal{N}}^{+}$it is known [9, Lemma $10($ ii) $]$ that $Z^{*} g(T) Z$ spectrally dominates $g\left(Z^{*} T Z\right)$. Hence, by Lemma 3.3,

$$
Z^{*} g(T) Z+\varepsilon I \geq W g\left(Z^{*} T Z\right) W^{*}
$$

for some unitary $W \in \mathcal{N}$. Then, by arguing as in the proof of [2, Theorem 2.1] or [8, Corollary 3.2], one can see that the claimed inequality holds for some unitaries $U, V \in \mathcal{N}$.

For the non-factor case, as in [16], we take the central direct integral decomposition into factors (see [27]) as

$$
\{\mathcal{N}, \mathcal{H}\}=\int_{\Omega}^{\oplus}\left\{\mathcal{N}_{\omega}, \mathcal{H}_{\omega}\right\} d \nu(\omega), \quad \tau=\int_{\Omega}^{\oplus} \tau_{\omega} d \nu(\omega)
$$

over a finite measure space $(\Omega, \mathcal{B}, \nu)$ that may be assumed to be complete. Then $A, B \in \mathcal{N}$ are represented as

$$
A=\int_{\Omega}^{\oplus} A_{\omega} d \nu(\omega), \quad B=\int_{\Omega}^{\oplus} B_{\omega} d \nu(\omega)
$$


with unique (a.e.) measurable fields $\omega \mapsto A_{\omega}, B_{\omega} \in \mathcal{N}_{\omega}$. For each $\omega \in \Omega$, from the first step of the proof, there are unitaries $U, V \in \mathcal{N}_{\omega}$ such that

$$
g\left(A_{\omega}+B_{\omega}\right)+\varepsilon I_{\omega} \geq U g\left(A_{\omega}\right) U^{*}+V g\left(B_{\omega}\right) V^{*} .
$$

Now, define $F(\omega)$ to be the set of pairs $(U, V)$ of unitaries in $\mathcal{N}_{\omega}$ satisfying (3.5), and prove that there are measurable fields $\omega \mapsto U_{\omega}$ and $\omega \mapsto V_{\omega}$ such that $\left(U_{\omega}, V_{\omega}\right) \in F(\omega)$ for all $\omega \in \Omega$. For this, as in [16], we may assume that $\omega \mapsto \mathcal{H}_{\omega}$ is a constant field $\mathcal{H}_{0}$. Then $F(\cdot)$ is a multifunction whose values are non-empty closed subsets of a Polish space $B\left(\mathcal{H}_{0}\right)_{1} \times B\left(\mathcal{H}_{0}\right)_{1}$, where $B\left(\mathcal{H}_{0}\right)_{1}$ is the closed unit ball of $B\left(\mathcal{H}_{0}\right)$ with the strong* topology. By using [20, Theorem 6.1], we infer that the graph

$$
\left\{(\omega, U, V) \in \Omega \times B\left(\mathcal{H}_{0}\right)_{1} \times B\left(\mathcal{H}_{0}\right)_{1}:(U, V) \in F(\omega)\right\}
$$

of $F(\cdot)$ belongs to $\mathcal{B} \otimes \mathcal{B}\left(B\left(\mathcal{H}_{0}\right)_{1} \times B\left(\mathcal{H}_{0}\right)_{1}\right)$, where $\mathcal{B}\left(B\left(\mathcal{H}_{0}\right)_{1} \times B\left(\mathcal{H}_{0}\right)_{1}\right)$ is the Borel $\sigma$-field of $B\left(\mathcal{H}_{0}\right)_{1} \times B\left(\mathcal{H}_{0}\right)_{1}$. Hence, as in [16] the measurable selection theorem (see e.g. [20]) yields measurable fields $\omega \mapsto U_{\omega}$ and $\omega \mapsto V_{\omega}$ as desired, so we obtain the claimed inequality with the unitaries $U=\int_{\Omega}^{\oplus} U_{\omega} d \nu(\omega)$ and $V=\int_{\Omega}^{\oplus} V_{\omega} d \nu(\omega)$ in $\mathcal{N}$.

Proof of Theorem 3.2. The matrix case is in [2, Theorem 2.1]. In the type $\mathrm{II}_{1}$ factor case, inequality (3.2) for a contraction $Z \in \mathcal{N}$ and an $T \in \overline{\mathcal{N}}^{+}$is, in turn, reversed as

$$
Z^{*} f(T) Z \leq W f\left(Z^{*} T Z\right) W^{*}+\varepsilon I
$$

by [9, Lemma 10(i)] and Lemma 3.3 similarly. (Here, note that although our assumption on $f$ is slightly weaker than that in [9], the proof of [9, Lemma 10(i)] can easily be modified to show that $f\left(Z^{*} T Z\right)$ spectrally dominates $Z^{*} f(T) Z$.) Hence the desired assertion follows in the factor case. Now, the proof for the non-factor case is the same as above.

An idea of the above proofs is to combine a unitary orbit technique with the measurable selection theorem. We end the section with another illustration of the idea, along the lines of [8, Proposition 2.11] for the matrix case.

Proposition 3.4. Let $g$ be a non-decreasing convex function on $[0, \infty)$. Assume that either $\mathcal{N}$ is a factor and $X, Y \in \overline{\mathcal{N}}$, or $X, Y \in \mathcal{N}$ without the factoriality of $\mathcal{N}$. Then, for every $\varepsilon>0$, there exist unitaries $U, V \in \mathcal{N}$ such that

$$
g(|X+Y|) \leq \frac{U g(|X|+|Y|) U^{*}+V g\left(\left|X^{*}\right|+\left|Y^{*}\right|\right) V^{*}}{2}+\varepsilon I .
$$


Proof. For any $X \in \overline{\mathcal{N}}$, the decomposition $X=\left|X^{*}\right|^{1 / 2} V|X|^{1 / 2}$ with a unitary $V$ shows that

$$
\left[\begin{array}{cc}
\left|X^{*}\right| & X \\
X^{*} & |X|
\end{array}\right]
$$

is a positive $\tau$-measurable operator affiliated with $\mathbb{M}_{2}(\mathcal{N})=\mathcal{N} \otimes \mathbb{M}_{2}$. Consequently,

$$
\left[\begin{array}{cc}
\left|X^{*}\right|+\left|Y^{*}\right| & X+Y \\
X^{*}+Y^{*} & |X|+|Y|
\end{array}\right]
$$

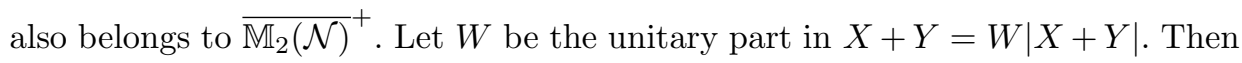

$$
\left[\begin{array}{ll}
-W^{*} & I
\end{array}\right]\left[\begin{array}{cc}
\left|X^{*}\right|+\left|Y^{*}\right| & X+Y \\
X^{*}+Y^{*} & |X|+|Y|
\end{array}\right]\left[\begin{array}{c}
-W \\
I
\end{array}\right]
$$

is in $\overline{\mathcal{N}}^{+}$, so that

$$
|X+Y| \leq \frac{|X|+|Y|+W^{*}\left(\left|X^{*}\right|+\left|Y^{*}\right|\right) W}{2}
$$

As $g$ is non-decreasing and convex, in the factor case, (3.6) combined with [13, Proposition 4.6(ii)] shows that $g(|X+Y|)$ is spectrally dominated by $\{g(|X|+|Y|)+$ $\left.W^{*} g\left(\left|X^{*}\right|+\left|Y^{*}\right|\right) W\right\} / 2$. Using Lemma 3.3 completes the proof of the proposition when $\mathcal{N}$ is a factor. The non-factor case follows by using the measurable selection method as in the previous proofs.

It is not known whether the measurable selection technique can work to prove Theorems 3.1, 3.2 or Proposition 3.4 when $A, B \in \overline{\mathcal{N}}^{+}$or $X, Y \in \overline{\mathcal{N}}$ without the factoriality of $\mathcal{N}$. But Theorems 3.1 and 3.2 will be applied to only bounded operators so that we shall never assume the factoriality of a finite von Neumann algebra in the rest of the paper.

\section{$\S 4$. Extension of symmetric norms and anti-norms}

The aim of this section is to show that a symmetric norm on $\mathcal{N}$ and a symmetric anti-norm on $\mathcal{N}^{+}$can naturally be extended, respectively, to $\overline{\mathcal{N}}$ and to $\overline{\mathcal{N}}^{+}$. First, let $\|\cdot\|$ be a symmetric norm on $\mathcal{N}$. For each $X \in \overline{\mathcal{N}}$ and $s>0$, the function $t \mapsto \beta_{s}(t):=\min \{s, t\}$ is used to define $|X| \wedge s:=\beta_{s}(|X|)$. Since Lemma 2.1 implies that $\||X| \wedge s\|$ is increasing as $s \nearrow \infty$, a natural extension of $\|\cdot\|$ to $\overline{\mathcal{N}}$ is given as

$$
\|X\|:=\lim _{s \nearrow \infty}\||X| \wedge s\|=\sup _{s>0}\||X| \wedge s\| \in[0, \infty]
$$


Proposition 4.1. The above extension of $\|\cdot\|$ becomes a symmetric norm on $\overline{\mathcal{N}}$ (with the value $\infty$ permitted).

Proof. It is immediate to see that the extended $\|\cdot\|$ on $\overline{\mathcal{N}}$ satisfies $\|\alpha X\|=|\alpha|\|X\|$ for all $\alpha \in \mathbb{C}$ and $\|U X V\|=\|X\|$ for all unitaries $U, V \in \mathcal{N}$. For every $A, B \in \overline{\mathcal{N}}^{+}$, $s>0$ and $\varepsilon>0$, since $\beta_{s}$ is concave on $[0, \infty)$, by Theorem 3.2 there are unitaries $U, V \in \mathcal{N}$ such that

$$
(A+B) \wedge s \leq U(A \wedge s) U^{*}+V(B \wedge s) V^{*}+\varepsilon I .
$$

By Lemma 2.1 this implies that

$$
\|(A+B) \wedge s\| \leq\|A \wedge s\|+\|B \wedge s\|+\varepsilon\|I\| .
$$

Letting $\varepsilon \searrow 0$ and $s \nearrow \infty$ gives $\|A+B\| \leq\|A\|+\|B\|$. Next we extend the monotonicity of $\|\cdot\|$ to $A \leq B$ in $\overline{\mathcal{N}}^{+}$. For every $s>0$ and $\varepsilon>0$ there exists a unitary $U \in \mathcal{N}$ such that $U(A \wedge s) U^{*} \leq B \wedge s+\varepsilon I$. Since $\lambda_{t}(A \wedge s) \leq \lambda_{t}(B \wedge s)$ for all $t \in(0, \tau(I))$, this follows from Lemma 3.3 when $\mathcal{N}$ is a factor. For the nonfactor case, we can use the measurable selection method under the central direct decomposition as in the previous section. Therefore, $\|A \wedge s\| \leq\|B \wedge s\|+\varepsilon\|I\|$, which implies that $\|A\| \leq\|B\|$. Now, the subadditivity $\|X+Y\| \leq\|X\|+\|Y\|$ in the whole $\overline{\mathcal{N}}$ follows from the triangle inequality

$$
|X+Y| \leq U|X| U^{*}+V|Y| V^{*}
$$

for some unitaries $U, V \in \mathcal{N}([13$, Lemma 4.3], [21]), or else by use of (3.6).

Secondly, let $\|\cdot\|$ ! be a symmetric anti-norm on $\mathcal{N}^{+}$. For every $A \in \overline{\mathcal{N}}^{+}$and

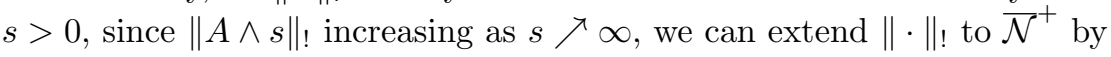

$$
\|A\| !:=\lim _{s \nearrow \infty}\|A \wedge s\| ! \in[0, \infty] .
$$

Proposition 4.2. The above extension of $\|\cdot\|$ ! to $\overline{\mathcal{N}}^{+}$still satisfies the three conditions $(1) !,(2)$ ! and $(3)$ !.

Proof. Since (1)! and (2)! are immediate by definition, we may prove (3)! . Let $A, B \in \overline{\mathcal{N}}^{+}, s>0$ and $\varepsilon>0$ be arbitrary. We show that there exists a unitary $U \in \mathcal{N}$ such that

$$
(A+B) \wedge 2 s+\varepsilon I \geq U(A \wedge s+B \wedge s) U^{*} .
$$

When $\mathcal{N}$ is a factor, this follows from Lemma 3.3 since we have, for $t \in(0, \tau(I))$, $\lambda_{t}((A+B) \wedge 2 s)=\lambda_{t}(A+B) \wedge 2 s \geq \lambda_{t}(A \wedge s+B \wedge s) \wedge 2 s=\lambda_{t}(A \wedge s+B \wedge s)$ 
due to $A \wedge s+B \wedge s \leq 2 s I$. For the non-factor case, under the decompositions (3.3) and (3.4) we have $A \wedge s=\int_{\Omega}^{\oplus} A_{\omega} \wedge s d \nu(\omega)$ and similarly for $B \wedge s$ and $(A+B) \wedge 2 s$. For each $\omega \in \Omega$, from the above factor case, there is a unitary $V \in \mathcal{N}_{\omega}$ such that

$$
\left(A_{\omega}+B_{\omega}\right) \wedge 2 s+\varepsilon I_{\omega} \geq V\left(A_{\omega} \wedge s+B_{\omega} \wedge s\right) V^{*}
$$

Now, define $F(\omega)$ to be the set of unitaries $V \in \mathcal{N}_{\omega}$ satisfying (4.2), and use the measurable selection method as before to obtain a measurable field $\omega \mapsto U_{\omega}$ such that $U_{\omega} \in F(\omega)$ for all $\omega \in \Omega$. Therefore, we have (4.1) with the unitary $U:=\int_{\Omega}^{\oplus} U_{\omega} d \nu(\omega)$ in $\mathcal{N}$, so that $\|(A+B) \wedge 2 s+\varepsilon I\|_{!} \geq\|A \wedge s\|_{!}+\|B \wedge s\|_{!}$. Letting $\varepsilon \searrow 0$ and $s \nearrow \infty$ gives $\|A+B\|_{!} \geq\|A\|_{!}+\|B\|_{!}$.

Remark 4.3. Let $\mathcal{N}=\bigoplus_{n=1}^{\infty} \mathcal{N}_{n}$ where $\mathcal{N}_{n}=\mathbb{M}_{2}$ for all $n$. We equip $\mathcal{N}$ with the trace $\tau=\sum_{n>1} 2^{-n} \tau_{n}$ where $\tau_{n}$ is the standard trace on $\mathcal{N}_{n}$. The function $t \mapsto \beta_{n}(t)=\min \{t, n\}$ is concave and there exist $A_{n}, B_{n} \in \mathbb{M}_{2}^{+}$such that $\operatorname{Tr} \beta_{n}\left(A_{n}+B_{n}\right)<\operatorname{Tr} \beta_{n}\left(A_{n}\right)+\operatorname{Tr} \beta_{n}\left(B_{n}\right)$. Hence, there exist $A, B \in \overline{\mathcal{N}}^{+}$such that $\tau((A+B) \wedge n))<\tau(A \wedge n)+\tau(A \wedge n)$ for all $n \in \mathbb{N}$. Such a phenomenon for $\tau$ (regarded as an anti-norm) explains why the proof of the superadditivity part (3)! in Proposition 4.2 is non-trivial.

Derived anti-norms extended to $\overline{\mathcal{N}}^{+}$have the following simple properties; (a) and (b) may be used to check that a symmetric anti-norm is not a derived one.

Proposition 4.4. Let $\|\cdot\|$ ! be a derived anti-norm on $\mathcal{N}^{+}$, which is derived from a symmetric norm $\|\cdot\|$ on $\mathcal{N}$ and a $p>0$. The following hold for the extensions of $\|\cdot\|$ ! to $\overline{\mathcal{N}}^{+}$and $\|\cdot\|$ to $\overline{\mathcal{N}}$ :

(a) $\|A\| !<\infty$ for all $A \in \overline{\mathcal{N}}^{+}$.

(b) If $A \in \overline{\mathcal{N}}^{+}$is singular, i.e., the kernel of $A$ is non-trivial, then $\|A\|_{!}=0$.

(c) If $A \in \overline{\mathcal{N}}^{+}$is non-singular, then $\|A\|_{\text {! }}=\left\|A^{-p}\right\|^{-1 / p}$ (with the convention $\left.\infty^{-1 / p}=0\right)$.

Proof. (a) is obvious once (b) and (c) have been proved.

(b) Assume that ker $A \neq\{0\}$, and let $P$ be the projection onto ker $A$. For each $s>0$ and $\varepsilon>0$ we have $(A \wedge s+\varepsilon I)^{-p} \geq \varepsilon^{-p} P$ and so $\left\|(A \wedge s+\varepsilon I)^{-p}\right\| \geq \varepsilon^{-p}\|P\|$. By definition (2.2),

$$
\|A \wedge s\|_{!}=\lim _{\varepsilon \searrow 0}\left\|(A \wedge s+\varepsilon I)^{-p}\right\|^{-1 / p} \leq \lim _{\varepsilon \searrow 0} \varepsilon\|P\|^{-1 / p}=0 .
$$

Therefore, $\|A\|_{!}=0$. 
(c) First we extend (2.2) to a non-singular $A \in \overline{\mathcal{N}}^{+}$. Let $A \in \overline{\mathcal{N}}^{+}$with $\operatorname{ker} A=\{0\}$; hence $A^{-p} \in \overline{\mathcal{N}}^{+}$. For every $s>0$ and $\varepsilon>0$, set

$$
\phi_{s}(\varepsilon):=\sup _{x \geq 0}\left\{x^{-p} \wedge s-(x+\varepsilon)^{-p}\right\} .
$$

Then it is clear that $\phi_{s}(\varepsilon)>0$ and $\lim _{\varepsilon \searrow 0} \phi_{s}(\varepsilon)=0$ for each $s>0$. Since $\left\|(A+\varepsilon I)^{-p}\right\|$ increases as $\varepsilon \searrow 0$ by Lemma 2.1 and

$$
A^{-p} \wedge s \leq(A+\varepsilon I)^{-p}+\phi_{s}(\varepsilon) I,
$$

we have $\left\|A^{-p} \wedge s\right\| \leq \lim _{\varepsilon \searrow 0}\left\|(A+\varepsilon I)^{-p}\right\|$. Hence $\left\|A^{-p}\right\| \leq \lim _{\varepsilon \searrow 0}\left\|(A+\varepsilon I)^{-p}\right\|$. On the other hand, since $(A+\varepsilon I)^{-p} \leq A^{-p} \wedge \varepsilon^{-p}$, we have $\left\|(A+\varepsilon I)^{-p}\right\| \leq\left\|A^{-p}\right\|$ for every $\varepsilon>0$. Therefore,

$$
\left\|A^{-p}\right\|^{-1 / p}=\lim _{\varepsilon \searrow 0}\left\|(A+\varepsilon I)^{-p}\right\|^{-1 / p} .
$$

Now, looking at the function $0 \leq x \mapsto(x \wedge s+\varepsilon)^{-p}$, one can easily see that

$$
(A+\varepsilon I)^{-p} \leq(A \wedge s+\varepsilon I)^{-p} \leq(A+\varepsilon I)^{-p}+s^{-p} I
$$

and hence

$$
\left\|(A+\varepsilon I)^{-p}\right\|^{-1 / p} \geq\left\|(A \wedge s+\varepsilon I)^{-p}\right\|^{-1 / p} \geq\left(\left\|(A+\varepsilon I)^{-p}\right\|+s^{-p}\|I\|\right)^{-1 / p} .
$$

Thanks to (4.3), letting $\varepsilon \searrow 0$ gives

$$
\left\|A^{-p}\right\|^{-1 / p} \geq\|A \wedge s\|_{!} \geq\left(\left\|A^{-p}\right\|+s^{-p}\|I\|\right)^{-1 / p}
$$

so that $\|A\|_{!}=\left\|A^{-p}\right\|^{-1 / p}$ follows.

\section{$\S 5$. Superadditivity for convex functions}

The aim of this section is to prove the next superadditivity theorem for a symmetric anti-norm involving a convex function $g$ on $[0, \infty)$. Note that a non-negative convex function $g$ on $[0, \infty)$ is superadditive if and only if $g(0)=0$. Also, note that the assumption on $g$ in the theorem is best possible; indeed, the assumption is necessary even for the classical Rotfel'd trace inequality for matrices.

Theorem 5.1. Let $A, B \in \overline{\mathcal{N}}^{+}$and let $g$ be a non-negative convex function on $[0, \infty)$ with $g(0)=0$. Then, for all symmetric anti-norms on $\mathcal{N}^{+}$,

$$
\|g(A+B)\|_{!} \geq\|g(A)\|_{!}+\|g(B)\|_{!}
$$


Here, and in what follows, we assume that symmetric anti-norms on $\mathcal{N}^{+}$are automatically defined on the full cone $\overline{\mathcal{N}}^{+}$as in Proposition 4.2, and similarly symmetric norms on $\mathcal{N}$ are defined on the whole $\overline{\mathcal{N}}$ as in Proposition 4.1.

Theorem 5.1 claims a numerical inequality; however, its proof relies on an operator inequality presented in Section 3. Indeed, when $A, B \in \mathcal{N}^{+}$, it is a straightforward consequence of Theorem 3.1. When $A, B \in \overline{\mathcal{N}}^{+}$in the factor case, we cannot argue by letting $\varepsilon \searrow 0$ in Theorem 3.1 because (4)! may not hold in $\overline{\mathcal{N}}^{+}$; for instance, when $\|\cdot\|$ ! is the Fuglede-Kadison determinant (see the end of Section 6), there is an $A \in \overline{\mathcal{N}}^{+}$which has the non-trivial kernel (hence $\|A\|_{!}=0$ ) but satisfies $\|A+\varepsilon I\|_{!}=\infty$ for all $\varepsilon>0$.

Proof of Theorem 5.1. Let $A, B \in \overline{\mathcal{N}}^{+}$and $s>0$. Since $g$ is continuous and non-decreasing on $[0, \infty)$, thanks to $[13$, Lemma $2.5($ iv) $]$ we have

$$
\begin{aligned}
\lambda_{t}(g((A+B) \wedge 2 s)) & =g\left(\lambda_{t}(A+B) \wedge 2 s\right) \\
& \geq g\left(\lambda_{t}(A \wedge s+B \wedge s)\right) \\
& =\lambda_{t}(g(A \wedge s+B \wedge s)), \quad t \in(0, \tau(I)) .
\end{aligned}
$$

Given $\varepsilon>0$, when $\mathcal{N}$ is a finite factor, Lemma 3.3 then entails

$$
g((A+B) \wedge 2 s)+\varepsilon I \geq W g(A \wedge s+B \wedge s) W^{*}
$$

for some unitary $W \in \mathcal{N}$. This inequality can be extended to the non-factor case by using the measurable selection method under the central direct decomposition as in Section 3; full details are left to the reader. Hence, Theorem 3.1 applied to $A \wedge s, B \wedge s$ in place of $A, B$ shows that

$$
g((A+B) \wedge 2 s)+2 \varepsilon I \geq U g(A \wedge s) U^{*}+V g(B \wedge s) V^{*}
$$

for some unitaries $U, V \in \mathcal{N}$. Therefore,

$$
\|g((A+B) \wedge 2 s)+2 \varepsilon I\|_{!} \geq\|g(A \wedge s)\|_{!}+\|g(B \wedge s)\|_{!}
$$

so that $\|g((A+B) \wedge 2 s)\|_{!} \geq\|g(A \wedge s)\|_{!}+\|g(B \wedge s)\|_{!}$. Since a simple estimation gives

$$
\|g(A)\|_{!}=\lim _{s \nearrow \infty}\|g(A) \wedge s\|_{!}=\lim _{s \nearrow \infty}\|g(A \wedge s)\|_{!}
$$

the claimed inequality follows.

In the rest of the section we collect a few special illustrations of Theorem 5.1. 
Corollary 5.2. Let $A, B \in \overline{\mathcal{N}}^{+}$be non-singular and $p_{1}, \ldots, p_{m}$ be positive scalars such that $\sum_{i=1}^{m} p_{i} \geq 1$. Then, for all symmetric norms on $\mathcal{N}$,

$$
\prod_{i=1}^{m}\left\|(A+B)^{-p_{i}}\right\|^{-1} \geq \prod_{i=1}^{m}\left\|A^{-p_{i}}\right\|^{-1}+\prod_{i=1}^{m}\left\|B^{-p_{i}}\right\|^{-1} .
$$

Proof. Let $g_{i}$ be strictly increasing convex functions on $[0, \infty)$ with $g_{i}(0)=0$, and let $q_{i}>0,1 \leq i \leq m$. Theorem 5.1 applied to the derived anti-norms $A \mapsto$ $\left\|A^{-q_{i}}\right\|^{-1 / q_{i}}$ yields, in view of Proposition 4.4(c),

$$
\left\|g_{i}^{-q_{i}}(A+B)\right\|^{-1 / q_{i}} \geq\left\|g_{i}^{-q_{i}}(A)\right\|^{-1 / q_{i}}+\left\|g_{i}^{-q_{i}}(B)\right\|^{-1 / q_{i}}, \quad 1 \leq i \leq m .
$$

Now, assume that $\sum_{i=1}^{m} q_{i}=1$. By the elementary inequality following from the concavity of the weighted geometric mean,

$$
\prod_{i=1}^{m}\left(a_{i}+b_{i}\right)^{q_{i}} \geq \prod_{i=1}^{m} a_{i}^{q_{i}}+\prod_{i=1}^{m} b_{i}^{q_{i}}
$$

with $a_{i}=\left\|g_{i}^{-q_{i}}(A)\right\|^{-1 / q_{i}}$ and $b_{i}=\left\|g_{i}^{-q_{i}}(B)\right\|^{-1 / q_{i}}$, we have

$$
\prod_{i=1}^{m}\left\|g_{i}^{-q_{i}}(A+B)\right\|^{-1} \geq \prod_{i=1}^{m}\left\|g_{i}^{-q_{i}}(A)\right\|^{-1}+\prod_{i=1}^{m}\left\|g_{i}^{-q_{i}}(B)\right\|^{-1} .
$$

Let $p=\sum_{i=1}^{m} p_{i}$. Take in (5.1) $g_{i}(t)=t^{p}$ and $q_{i}=p_{i} / p, 1 \leq i \leq m$, to obtain the required estimate.

Corollary 5.3. Let $A, B \in \overline{\mathcal{N}}^{+}$be non-singular. Then, for all symmetric norms on $\mathcal{N}$, and $m=1,2, \ldots$,

$$
\left\|\sum_{k=1}^{m}(A+B)^{-k}\right\|^{-1} \geq\left\|\sum_{k=1}^{m} A^{-k}\right\|^{-1}+\left\|\sum_{k=1}^{m} B^{-k}\right\|^{-1} .
$$

Proof. Let $m \geq 2$ and let

$$
g(t):=\frac{t^{m}}{1+t+\cdots+t^{m-1}}=\frac{t^{m+1}-t^{m}}{t^{m}-1} .
$$

Then $g(0)=0$ and for $t>0$,

$$
g^{\prime \prime}(t)=\frac{m t^{m-2}\left\{(m-1) t^{m+1}-(m+1) t^{m}+(m+1) t-(m-1)\right\}}{\left(t^{m}-1\right)^{3}} .
$$

For $\phi(t):=(m-1) t^{m+1}-(m+1) t^{m}+(m+1) t-(m-1)$ compute

$$
\begin{aligned}
\phi^{\prime}(t) & =(m+1)\left\{(m-1) t^{m}-m t^{m-1}+1\right\}, \\
\phi^{\prime \prime}(t) & =(m+1) m(m-1) t^{m-2}(t-1) .
\end{aligned}
$$


Since $\phi(1)=\phi^{\prime}(1)=\phi^{\prime \prime}(1)=0$, we see that $\phi(t) \leq 0$ for $0 \leq t \leq 1$ and $\phi(t) \geq 0$ for $t \geq 1$. Hence $g^{\prime \prime}(t) \geq 0$ for all $t>0$, so $g(t)$ is convex on $(0, \infty)$. Note that $g(t)=\left(\sum_{k=1}^{m} t^{-k}\right)^{-1}$ for $t>0$. So, applying Theorem 5.1 to this $g$ and the derived anti-norm $A \mapsto\left\|A^{-1}\right\|^{-1}$ proves the corollary.

The next corollary involves an anti-norm specific to the matrix algebra $\mathbb{M}_{n}$. Here $\bigwedge^{m} A$ denotes the $m$ th antisymmetric tensor power of a matrix $A$ (see [4]).

Corollary 5.4. Let $A, B \in \mathbb{M}_{n}^{+}$be non-singular. Let $g:[0, \infty) \rightarrow[0, \infty)$ be a strictly increasing convex function with $g(0)=0$. Then, for all $0<q \leq 1$ and all $m=1,2, \ldots$,

$$
\left\{\frac{\operatorname{Tr} \bigwedge^{m} g^{q}(A+B)}{\operatorname{Tr} \bigwedge^{m-1} g^{q}(A+B)}\right\}^{1 / q} \geq\left\{\frac{\operatorname{Tr} \bigwedge^{m} g^{q}(A)}{\operatorname{Tr} \bigwedge^{m-1} g^{q}(A)}\right\}^{1 / q}+\left\{\frac{\operatorname{Tr} \bigwedge^{m} g^{q}(B)}{\operatorname{Tr} \bigwedge^{m-1} g^{q}(B)}\right\}^{1 / q} .
$$

Note that letting $m=q=1$ we recapture the Rotfel'd trace inequality.

Proof. By a theorem of Marcus and Lopes [24] (also [25, p. 116]), the functional on positive non-singular matrices

$$
A \mapsto \frac{\operatorname{Tr} \bigwedge^{m} A}{\operatorname{Tr} \bigwedge^{m-1} A}
$$

is superadditive. This can be extended as an anti-norm on the whole of $\mathbb{M}_{n}^{+}$by using condition (4)! . The corollary then follows from Lemma 2.8 combined with Theorem 5.1.

\section{$\S 6$. Full symmetry and majorization}

In this section we consider a stronger symmetry property of norms and anti-norms in connection with majorization relations. We will focus on the case of diffuse algebras. Indeed, the case of $\mathbb{M}_{n}$ is simpler as well as classical. Meanwhile, the setting of a general finite von Neumann algebra $\mathcal{N}$ is inappropriate to apply the majorization technique. This may be justified by the fact [14, Theorem 3.27] that $(\mathcal{N}, \tau)$ with $\tau(I)=1$ satisfies the weak Dixmier property (i.e., $\tau(A)$ is in the $\|\cdot\|_{\infty^{-}}$ closure of the convex hull of $\left\{B \in \mathcal{N}^{+}: \lambda(B)=\lambda(A)\right\}$ for every $A \in \mathcal{N}^{+}$) if and only if either $(\mathcal{N}, \tau)$ is a subalgebra of $\left(\mathbb{M}_{n}, n^{-1} \mathrm{Tr}\right)$ containing all diagonal matrices, or $\mathcal{N}$ is diffuse.

Thus, in Sections 6 and 7, we shall always use $\mathcal{M}$ (but not $\mathcal{N}$ ) to denote a diffuse finite von Neumann algebra with a faithful normal trace $\tau$ such that $\tau(I)=1$. 
Definition 6.1. A symmetric norm $\|\cdot\|$ on $\mathcal{M}$ is said to be fully symmetric (or rearrangement invariant) if $\lambda(A)=\lambda(B)$ implies $\|A\|=\|B\|$ for $A, B \in \mathcal{M}^{+}$. Also, a symmetric anti-norm $\|\cdot\|$ ! on $\mathcal{M}^{+}$is said to be fully symmetric if the same holds for $\|\cdot\|$ !

The next proposition says that symmetry and full symmetry are equivalent when $\mathcal{M}$ is a $\mathrm{II}_{1}$ factor (this is also true and well-known for $\mathbb{M}_{n}$ ).

Proposition 6.2. If $\mathcal{M}$ is a factor, then any symmetric norm and any symmetric anti-norm are fully symmetric.

Proof. Let $A, B \in \mathcal{M}^{+}$and assume that $\lambda(A)=\lambda(B)$. For a symmetric norm $\|\cdot\|$, by Lemmas 2.1 and 3.3 we have $\|A\| \leq\|B\|+\varepsilon\|I\|$ for every $\varepsilon>0$, so $\|A\| \leq\|B\|$, and similarly for the reverse inequality. The proof is similar for a symmetric anti-norm by using condition (4)!

Recall some notions of majorization relevant to our discussion below. For $A, B \in \overline{\mathcal{M}}^{+}$, the submajorization $A \prec_{w} B$ is defined as $\lambda(A) \prec_{w} \lambda(B)$, i.e.,

$$
\int_{0}^{t} \lambda_{s}(A) d s \leq \int_{0}^{t} \lambda_{s}(B) d s, \quad t \in(0,1),
$$

and the majorization $A \prec B$ means that $A \prec w B$ and $\tau(A)=\tau(B)<\infty$. The supermajorization $A \prec{ }^{w} B$ is defined as

$$
\int_{t}^{1} \lambda_{s}(A) d s \geq \int_{t}^{1} \lambda_{s}(B) d s, \quad t \in(0,1) .
$$

The log-supermajorization $A \prec^{w(\log )} B$ is defined as

$$
\int_{t}^{1} \log \lambda_{s}(A) d s \geq \int_{t}^{1} \log \lambda_{s}(B) d s, \quad t \in(0,1) .
$$

These definitions make sense since the integrals always exist, with $\pm \infty$ permitted. (The finiteness assumption $\tau(I)<\infty$ is essential to introduce supermajorization and log-supermajorization.)

Example 6.3. (1) For each $t \in(0,1]$ the functional

$$
\|X\|_{(t)}:=\int_{0}^{t} \mu_{s}(X) d s, \quad X \in \mathcal{M},
$$

is a fully symmetric norm on $\mathcal{M}$, which is the continuous version of the Ky Fan $k$-norm for matrices. The triangle inequality for $\|\cdot\|_{(t)}$ is a consequence of the submajorization $\mu(X+Y) \prec_{w} \mu(X)+\mu(Y)$ for $X, Y \in \overline{\mathcal{M}}$ (see [17]). 
(2) For each $t \in(0,1]$ the functional

$$
\|A\|_{\{t\}}:=\int_{1-t}^{1} \lambda_{s}(A) d s, \quad A \in \mathcal{M}^{+},
$$

is a fully symmetric anti-norm on $\mathcal{M}^{+}$. The superadditivity of $\|\cdot\|_{\{t\}}$ is a consequence of $\lambda(A+B) \prec \lambda(A)+\lambda(B)$ for $A, B \in \mathcal{M}^{+}$(see [17]). This anti-norm is not a derived anti-norm.

(3) For each $t \in(0,1]$ and $p>0$, the anti-norm derived from the above $\|\cdot\|_{(t)}$ and $p$ is written as

$$
\|A\|_{!}:=\lim _{\varepsilon \searrow 0}\left\|(A+\varepsilon I)^{-p}\right\|_{(t)}^{-1 / p}=\left(\int_{1-t}^{1} \lambda_{s}(A)^{-p} d s\right)^{-1 / p}, \quad A \in \mathcal{M}^{+},
$$

with the usual convention $0^{-p}=\infty$ and $\infty^{-1 / p}=0$. Obviously, this anti-norm is fully symmetric. One can easily find a sequence $A_{n}, A \in \mathcal{M}^{+}$such that $\left\|A_{n}-A\right\|_{\infty}$ $\rightarrow 0$ and $\left\|A_{n}\right\|_{!}=0$ for all $n$, but $\|A\|_{!}>0$. Therefore, $\|\cdot\| !$ is not $\|\cdot\|_{\infty}$-continuous on $\mathcal{M}^{+}$. On the other hand, the anti-norm derived from $\|\cdot\|_{\infty}$ (and any $p>0$ ) is $\lambda_{1}(A)$, which is $\|\cdot\|_{\infty}$-continuous on $\mathcal{M}^{+}$. Thus, the continuity behavior with respect to the operator norm in the diffuse case is subtler than in the matrix case.

Since $\mathcal{M}$ is diffuse, we can choose a family $\left\{F_{t}\right\}_{0 \leq t \leq 1}$ of projections as in the proof of Lemma 3.3. In our discussion below we will use such a family $\left\{F_{t}\right\}$ without explicit mention.

\section{§6.1. Fully symmetric norms}

The following properties of fully symmetric norms were discussed in more or less detail in the study of non-commutative Banach function spaces (see e.g. [11, 12, 28]), usually as working assumptions rather than results. The book [23] contains a nice discussion of this topic.

Proposition 6.4. Let $\|\cdot\|$ be a fully symmetric norm on $\mathcal{M}$.

(a) For every $A, B \in \overline{\mathcal{M}}^{+}, A \prec_{w} B$ implies $\|A\| \leq\|B\|$.

(b) If $A, A_{n} \in \overline{\mathcal{M}}^{+}$and $A_{n} \nearrow A$ in the $\tau$-measure topology (or more weakly $\lambda_{t}\left(A_{n}\right) \nearrow \lambda_{t}(A)$ for a.e. $\left.t \in(0,1)\right)$, then $\left\|A_{n}\right\| \nearrow\|A\|$.

(c) $\|X\|_{1}\|I\| \leq\|X\| \leq\|X\|_{\infty}\|I\|$ for all $X \in \overline{\mathcal{M}}$, where $\|X\|_{1}:=\tau(|X|)$ and $\|X\|_{\infty}:=\lim _{t \searrow 0} \lambda_{t}(|X|)$, the operator norm.

Proof. (a) (for bounded operators) Assume that $A, B \in \mathcal{M}^{+}$and $A \prec_{w} B$. Since $f:=\lambda(A) \prec_{w} g:=\lambda(B)$ as functions in $L^{\infty}(0,1)^{+}$, by [10, Theorem 1.1] there is an $h \in L^{\infty}(0,1)^{+}$such that $f \leq h \prec g$. Let $\Gamma$ denote the set of bijective Borel 
transformations on $(0,1)$ preserving the Lebesgue measure. As in the proof of [19, Theorem 2.1, Lemma 2.2] we can see that the decreasing rearrangement $h^{*}$ of $h$ is in the $\|\cdot\|_{\infty}$-closure of the convex hull of $\{\gamma f: \gamma \in \Gamma\}$, where $(\gamma f)(t):=f\left(\gamma^{-1} t\right)$. Thus, for every $\varepsilon>0$ there are $\gamma_{i} \in \Gamma$ and $\lambda_{i}>0,1 \leq i \leq k$, such that $\sum_{i=1}^{k} \lambda_{i}=1$ and $h^{*} \leq \sum_{i=1}^{k} \lambda_{i} \gamma_{i} g+\varepsilon 1$. Let $C:=\int_{0}^{1} h^{*}(t) d F_{t}$ and $B_{i}:=\int_{0}^{1}\left(\gamma_{i} g\right)(t) d F_{t}$. Since $\lambda\left(B_{i}\right)=\lambda(B)$, the monotonicity and full symmetry of $\|\cdot\|$ yield

$$
\|A\| \leq\|C\| \leq \sum_{i=1}^{k} \lambda_{i}\left\|B_{i}\right\|+\varepsilon\|I\|=\|B\|+\varepsilon\|I\| .
$$

Hence $\|A\| \leq\|B\|$.

(b) First we prove that if $A, A_{n} \in \mathcal{M}^{+}$and $\lambda_{t}\left(A_{n}\right) \nearrow \lambda_{t}(A)$ a.e., then $\left\|A_{n}\right\| \nearrow\|A\|$. By (a), $\left\|A_{n}\right\|$ is increasing in $n$. For every $\varepsilon>0$, since $\lambda_{0}\left(A_{n}\right)=$ $\left\|A_{n}\right\|_{\infty} \nearrow \lambda_{0}(A)=\|A\|_{\infty}$ and

$$
\lim _{t \searrow 0} \frac{1}{t} \int_{0}^{t}\left(\lambda_{s}(A)-\lambda_{s}\left(A_{n}\right)\right) d s=\lambda_{0}(A)-\lambda_{0}\left(A_{n}\right),
$$

one can choose $n_{0} \in \mathbb{N}$ and $\delta>0$ so that $t^{-1} \int_{0}^{t}\left(\lambda_{s}(A)-\lambda_{s}\left(A_{n_{0}}\right)\right) d s<\varepsilon$ for all $t \in(0, \delta)$, and hence

$$
\frac{1}{t} \int_{0}^{t}\left(\lambda_{s}(A)-\lambda_{s}\left(A_{n}\right)\right) d s<\varepsilon, \quad t \in(0, \delta), n \geq n_{0}
$$

Furthermore, for any $t \in[\delta, 1)$ one has

$$
\frac{1}{t} \int_{0}^{t}\left(\lambda_{s}(A)-\lambda_{s}\left(A_{n}\right)\right) d s \leq \frac{1}{\delta} \int_{0}^{1}\left(\lambda_{s}(A)-\lambda_{s}\left(A_{n}\right)\right) d s \quad \searrow \quad \text { as } n \rightarrow \infty
$$

by the dominated convergence theorem. Hence there exists an $n_{1} \in \mathbb{N}$ such that

$$
\frac{1}{t} \int_{0}^{t}\left(\lambda_{s}(A)-\lambda_{s}\left(A_{n}\right)\right) d s<\varepsilon, \quad t \in[\delta, 1), n \geq n_{1} .
$$

If $n \geq \max \left\{n_{0}, n_{1}\right\}$, then the above estimates imply that $A \prec_{w} A_{n}+\varepsilon I$ so that $\|A\| \leq\left\|A_{n}\right\|+\varepsilon\|I\|$ by (a). Thus $\left\|A_{n}\right\| \nearrow\|A\|$.

Next assume that $A, A_{n} \in \overline{\mathcal{M}}^{+}$and $\lambda_{t}\left(A_{n}\right) \nearrow \lambda_{t}(A)$ a.e. For every $s>0$, since $\lambda_{t}\left(A_{n} \wedge s\right) \nearrow \lambda_{t}(A \wedge s)$ a.e., we have $\left\|A_{n} \wedge s\right\| \nearrow\|A \wedge s\|$ from the first step. Hence $\left\|A_{n}\right\|=\sup _{s>0}\left\|A_{n} \wedge s\right\|$ is increasing in $n$ and

$$
\|A\|=\sup _{s>0}\|A \wedge s\|=\sup _{s>0, n \in \mathbb{N}}\left\|A_{n} \wedge s\right\|=\sup _{n \in \mathbb{N}}\left\|A_{n}\right\| .
$$

Therefore, $\left\|A_{n}\right\| \nearrow\|A\|$. 
(c) For $X \in \mathcal{M}$, the inequality $\|X\| \leq\|X\|_{\infty}\|I\|$ was given in (2.1). Since $\tau(|X|) I \prec|X|$, we have $\|X\|_{1}\|I\| \leq\|X\|$ by (a). These easily extend to all $X \in \overline{\mathcal{M}}$ by $(\mathrm{b})$.

(a) (for unbounded operators) Let $A, B \in \overline{\mathcal{M}}^{+}$and assume $A \prec_{w} B$. We may assume that $\|B\|<\infty$ and so $\|A\|_{1} \leq\|B\|_{1}<\infty$ by (c). Fix $0<\rho<1$. Then, for each $n \in \mathbb{N}$, since $\int_{0}^{t} \lambda_{s}(B) \wedge m d s \nearrow \int_{0}^{t} \lambda_{s}(B) d s$ as $m \rightarrow \infty$ uniformly in $t \in(0,1)$, one can choose an $m \in \mathbb{N}$ such that $\rho A \wedge n \prec_{w} B \wedge m$ and hence $\|\rho A \wedge n\| \leq\|B\|$ by (a). Hence $\|A\| \leq\|B\|$ by letting $n \rightarrow \infty$ and then $\rho \nearrow 1$.

In view of Example 6.3(1) we have

Corollary 6.5. Let $X, Y \in \overline{\mathcal{M}}$. Then $|X| \prec_{w}|Y|$ (i.e., $\left.\mu(X) \prec_{w} \mu(Y)\right)$ if and only if $\|X\| \leq\|Y\|$ for all fully symmetric norms on $\mathcal{M}$ (extended to $\overline{\mathcal{M}}$ ).

\section{$\S 6.2$. Fully symmetric anti-norms}

Fully symmetric anti-norms have the following properties. It would be worthwhile considering these properties in parallel to those in Proposition 6.4.

Proposition 6.6. Let $\|\cdot\|$ ! be a fully symmetric anti-norm on $\mathcal{M}^{+}$.

(a) For every $A, B \in \overline{\mathcal{M}}^{+}, A \prec^{w} B$ implies $\|A\|_{!} \geq\|B\|_{\text {! }}$

(b) If $A, A_{n} \in \mathcal{M}^{+}$and $A_{n} \searrow A$ (or more weakly $\lambda_{t}\left(A_{n}\right) \searrow \lambda_{t}(A)$ for a.e.

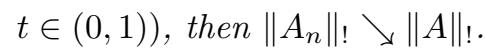

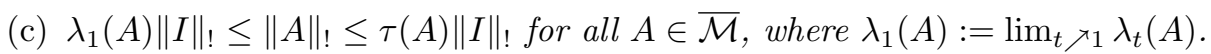

Proof. (a) Since $A \prec^{w} B$ implies $A \wedge s \prec^{w} B \wedge s$ for any $s>0$ (which can be seen as in the discrete case [25, p. 167]), we may assume $A, B \in \mathcal{M}^{+}$. Since $f:=\lambda(A) \prec^{w} g:=\lambda(B)$ as functions in $L^{\infty}(0,1)^{+}$, there is an $h \in L^{\infty}(0,1)^{+}$ such that $f \geq h \prec g$. The remaining proof being similar to that of Proposition 6.4(a), we omit the details.

(b) Assume that $A, A_{n} \in \mathcal{M}$ and $\lambda_{t}\left(A_{n}\right) \searrow \lambda_{t}(A)$ for a.e. $t \in(0,1)$. By (a),

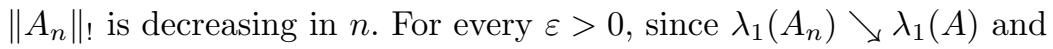

$$
\lim _{t \searrow 0} \frac{1}{t} \int_{1-t}^{1}\left(\lambda_{s}\left(A_{n}\right)-\lambda_{s}(A)\right) d s=\lambda_{1}\left(A_{n}\right)-\lambda_{1}(A),
$$

one can show that $A+\varepsilon I \prec^{w} A_{n}$ for all sufficiently large $n$ as in the proof of Proposition 6.4(b) by replacing $t^{-1} \int_{0}^{t}$ with $t^{-1} \int_{1-t}^{1}$. By (a) this implies $\|A+\varepsilon I\|_{!} \geq$ $\lim _{n}\left\|A_{n}\right\|_{\text {! }}$. Letting $\varepsilon \searrow 0$ gives $\|A\|_{!} \geq \lim _{n}\left\|A_{n}\right\|_{\text {! }}$ and so $\left\|A_{n}\right\|_{\text {! }} \searrow\|A\|_{\text {! }}$.

(c) For $A \in \overline{\mathcal{M}}^{+}, \lambda_{1}(A) I \leq A$ implies that $\lambda_{1}(A)\|I\|_{\text {! }} \leq\|A\|_{\text {! }}$. Assuming $\tau(A)<\infty$, we have $\tau(A)\|I\|_{\text {! }} \geq\|A\|_{\text {! from }} \tau(A) I \prec A$.

In view of Example 6.3(2) we have 
Corollary 6.7. Let $A, B \in \overline{\mathcal{M}}^{+}$. Then $A \prec^{w} B$ if and only if $\|A\| ! \leq\|B\|$ ! for all fully symmetric anti-norms on $\mathcal{M}^{+}$(extended to $\overline{\mathcal{M}}^{+}$).

Remark 6.8. Proposition 6.4(b) means that a fully symmetric norm extended to $\overline{\mathcal{M}}$ satisfies the Fatou property (see [12]). Proposition 6.6(b) is considered as the "anti-Fatou property". Even though (4)! may not hold in $\overline{\mathcal{M}}^{+}$as noted in Section 5 , it is not known whether the anti-Fatou property holds for $A, A_{n} \in \overline{\mathcal{M}}^{+}$ when $\left\|A_{n}\right\|_{!}<\infty$ and $A_{n} \searrow A$ in the $\tau$-measure topology. For fully symmetric derived anti-norms, this property will be shown in the next subsection.

\section{§6.3. Fully symmetric derived anti-norms}

In the rest of the section we will consider fully symmetric derived anti-norms.

Lemma 6.9. Let $\|\cdot\|_{!}$be an anti-norm on $\mathcal{M}^{+}$which is derived from a symmetric norm $\|\cdot\|$ on $\mathcal{M}$ and a $p>0$. Then $\|\cdot\| !$ is fully symmetric if and only if so is $\|\cdot\|$.

Proof. From (2.1), a symmetric norm on $\mathcal{M}$ is fully symmetric if the condition in Definition 6.1 holds for invertible $A, B \in \mathcal{M}^{+}$. From condition (4)! the same is true for a symmetric anti-norm. Hence the assertion follows from the relations $\|A\|_{!}=\left\|A^{-p}\right\|^{-1 / p}$ and $\|A\|=\left\|A^{-1 / p}\right\|_{!}^{-p}$ for invertible $A \in \mathcal{M}^{+}$.

Properties (a) and (b) of Proposition 6.6 are strengthened for fully symmetric derived anti-norms as follows. Note that $A \prec^{w(\log )} B$ is weaker than $A \prec^{w} B$ for $A, B \in \overline{\mathcal{M}}^{+}$.

Proposition 6.10. Let $\|\cdot\| !$ be a fully symmetric derived anti-norm on $\mathcal{M}^{+}$.

(a) For every $A, B \in \overline{\mathcal{M}}^{+}, A \prec w(\log ) B$ implies $\|A\|_{!} \geq\|B\|_{\text {! }}$.

(b) If $A, A_{n} \in \overline{\mathcal{M}}^{+}$and $A_{n} \searrow A$ in the $\tau$-measure topology (or more weakly $\lambda_{t}\left(A_{n}\right) \searrow \lambda_{t}(A)$ for a.e. $\left.t \in(0,1)\right)$, then $\left\|A_{n}\right\|_{!} \searrow\|A\|_{!}$. In particular, (4)! holds in $\overline{\mathcal{M}}^{+}$.

Proof. From Lemma 6.9 , let $\|\cdot\|$ ! be derived from a fully symmetric norm $\|\cdot\|$ and a $p>0$.

(a) Assume that $A \prec^{w(\log )} B$. Since this implies that $A \wedge s \prec^{w(\log )} B \wedge s$ for all $s>0$ (similarly to the assertion for $\prec^{w}$ in the proof of Proposition 6.6, by considering the function $\left.\log \left(e^{x} \wedge s\right)\right)$, we can assume that $A, B \in \mathcal{M}^{+}$. Furthermore, by replacing $A$ with $A+\varepsilon I$ for any $\varepsilon>0, A$ may be assumed to be invertible. First, we assume that $\int_{0}^{1} \log \lambda_{s}(B) d s=-\infty$, and we prove that $\|B\| !=0$ for every derived anti-norm $\|\cdot\| !$. By Proposition 6.4(c) it suffices to prove this for the anti-norm derived from $\|\cdot\|_{1}$ and any $p>0$. So we may show that $\lim _{\varepsilon \searrow 0}\left\|(B+\varepsilon I)^{-p}\right\|_{1}=\infty$. Since $\int_{0}^{1} \log \lambda_{s}(B) d s=-\infty$ implies that $\lambda_{1}(B)=0$, 
we have $-\log \lambda_{s}(B) \leq \lambda_{s}(B)^{-p}$ for all $s$ sufficiently near 1 . Hence by (6.1) for $t=1$,

$$
\lim _{\varepsilon \searrow 0}\left\|(B+\varepsilon I)^{-p}\right\|_{1}=\int_{0}^{1} \lambda_{s}(B)^{-p} d s=\infty .
$$

Next assume that $B$ as well as $A$ are invertible. Then $A \prec^{w(\log )} B$ means $\log \lambda(A) \prec^{w} \log \lambda(B)$. For every $p>0$, since $\log \lambda\left(A^{-p}\right) \prec_{w} \log \lambda\left(B^{-p}\right)$, we have $A^{-p} \prec_{w} B^{-p}$ by [17, Proposition 1.2]. Hence by Proposition $6.4(\mathrm{a}),\left\|A^{-p}\right\| \leq$ $\left\|B^{-p}\right\|$ for any fully symmetric norm $\|\cdot\|$, implying the assertion.

Finally, assume that $B$ is not invertible but $\int_{0}^{1} \log \lambda_{s}(B) d s>-\infty$, so $\lambda_{s}(B)>0$ for all $s \in(0,1)$ and $\log \lambda(B)$ is integrable on $(0,1)$. One can fix an $r_{0} \in(0,1)$ such that $\lambda_{r_{0}}(B) \leq \lambda_{1}(A)$ (since $A$ is invertible while $B$ is not). For every $\delta>0$ there exists an $r \in\left(r_{0}, 1\right)$ such that

$$
\int_{r}^{1}\left(\log \lambda_{r}(B)-\log \lambda_{s}(B)\right) d s \leq \delta\left(1-r_{0}\right)
$$

so we define

$$
\hat{B}:=\int_{0}^{r} \lambda_{s}(B) d F_{s}+\int_{r}^{1} \lambda_{r}(B) d F_{s},
$$

which is invertible. If $t \in\left(r_{0}, 1\right)$, then

$$
\int_{t}^{1}\left(\log \lambda_{s}(A)+\delta\right) d s \geq \int_{t}^{1} \log \lambda_{s}(A) d s \geq \int_{t}^{1} \log \lambda_{r_{0}}(B) d s \geq \int_{t}^{1} \log \lambda_{s}(\hat{B}) d s .
$$

If $t \in\left(0, r_{0}\right]$, then

$$
\begin{aligned}
\int_{t}^{1}\left(\log \lambda_{s}(A)+\delta\right) d s & \geq \int_{t}^{1} \log \lambda_{s}(B) d s+\delta\left(1-r_{0}\right) \\
& =\int_{t}^{1} \log \lambda_{s}(\hat{B}) d s-\int_{r}^{1}\left(\log \lambda_{r}(B)-\log \lambda_{s}(B)\right) d s+\delta\left(1-r_{0}\right) \\
\geq & \int_{t}^{1} \log \lambda_{s}(\hat{B}) d s .
\end{aligned}
$$

The above estimates imply that $e^{\delta} A \prec^{w(\log )} \hat{B}$. Since $\hat{B}$ is invertible, $\left\|\left(e^{\delta} A\right)^{-p}\right\| \leq$ $\left\|\hat{B}^{-p}\right\|$ as in the previous case. Therefore,

$$
e^{\delta}\left\|A^{-p}\right\|^{-1 / p} \geq\left\|\hat{B}^{-p}\right\|^{-1 / p}=\|\hat{B}\|_{!} \geq\|B\| !
$$

by Proposition $6.6(\mathrm{a})$ since $\lambda(\hat{B}) \geq \lambda(B)$. Letting $\delta \searrow 0$ gives $\|A\|_{!} \geq\|B\|_{\text {! }}$.

(b) If $\operatorname{ker} A \neq\{0\}$, then there is a $\delta \in(0,1)$ such that $\lambda_{1-\delta}(A)=0$. Since $\lambda_{1-\delta / 2}\left(A_{n}\right) \searrow 0$, for every $\varepsilon>0$ there exists an $n_{0} \in \mathbb{N}$ such that $\lambda_{1-\delta / 2}\left(A_{n}\right)<\varepsilon$ 
for all $n \geq n_{0}$. For each $n \geq n_{0}$, letting $P_{n}:=\mathbf{1}_{[0, \varepsilon]}\left(A_{n}\right)$ we have $\tau\left(P_{n}\right) \geq \delta / 2$ and $\left(A_{n}+\varepsilon I\right)^{-p} \geq(2 \varepsilon)^{-p} P_{n}$. Therefore,

$$
\left\|\left(A_{n}+\varepsilon I\right)^{-p}\right\| \geq(2 \varepsilon)^{-p}\left\|P_{n}\right\| \geq(2 \varepsilon)^{-p}\|I\| \delta / 2
$$

so that $\left\|A_{n}\right\|_{!} \leq 2 \varepsilon(\|I\| \delta / 2)^{-1 / p}$ for all $n \geq n_{0}$. Hence $\left\|A_{n}\right\|_{!} \searrow 0$.

Next assume that $\operatorname{ker} A=\{0\}$, so $A^{-p} \in \overline{\mathcal{M}}^{+}$. Since

$$
\lambda_{t}\left(A_{n}^{-p}\right)=\lambda_{1-t}\left(A_{n}\right)^{-p} \nearrow \lambda_{1-t}(A)^{-p}=\lambda_{t}\left(A^{-p}\right)
$$

for a.e. $t \in(0,1)$, we have $\left\|A_{n}^{-p}\right\| \nearrow\left\|A^{-p}\right\|$ by Proposition 6.4(b). Therefore, $\left\|A_{n}\right\|_{!} \searrow\|A\|$ ! by Proposition 4.4(c).

The next theorem is concerned with the converse to Proposition 6.10(a).

Theorem 6.11. Let $A, B \in \overline{\mathcal{M}}^{+}$and assume that $\int_{0}^{1} \lambda_{s}(B)^{-p} d s<\infty$ for some $p>0$ (in particular, this is the case if $B \geq \delta I$ for some $\delta>0$ ). Then the following two conditions are equivalent:

(i) $A \prec w(\log ) B$.

(ii) $\|A\| ! \geq\|B\|$ ! for every fully symmetric derived anti-norm $\|\cdot\|$ ! on $\mathcal{M}^{+}$.

To prove the theorem, we first give a lemma. When $A$ is invertible, the lemma is [3, Lemma 4.3.6] with a simpler proof.

Lemma 6.12. Let $A \in \mathcal{M}^{+}$and assume that $\int_{0}^{1} \lambda_{s}(A)^{-p} d s<\infty$ for some $p>0$. Then, for every $t \in(0,1]$,

$$
\exp \left(\frac{1}{t} \int_{1-t}^{1} \log \lambda_{s}(A) d s\right)=\lim _{p \searrow 0}\left(\frac{1}{t} \int_{1-t}^{1} \lambda_{s}(A)^{-p} d s\right)^{-1 / p}=\lim _{p \searrow 0} t^{1 / p}\left\|A^{-p}\right\|_{(t)}^{-1 / p},
$$

where $\|\cdot\|_{(t)}$ is as in Example 6.3(1).

Proof. Replacing $A$ with $\alpha A$ for some $\alpha>0$, we may suppose that $A \leq I$. Assume that $\int_{0}^{1} \lambda_{s}(A)^{-p_{0}} d s<\infty$ for some $p_{0}>0$; then $0<\lambda_{s}(A) \leq 1$ for all $s \in(0,1)$ and $-\lambda_{s}(A)^{-p} \log \lambda_{s}(A)$ is integrable on $(0,1)$ for every $p \in\left(0, p_{0}\right)$. Write $\varphi(s, p):=$ $\lambda_{s}(A)^{-p}$ for $s \in(0,1)$ and $p \in\left(0, p_{0}\right)$. Since

$$
\frac{\varphi(s, p)-\varphi(s, 0)}{p}=\partial_{p} \varphi(s, \theta p)=-\lambda_{s}(A)^{-\theta p} \log \lambda_{s}(A) \leq-\lambda_{s}(A)^{-p} \log \lambda_{s}(A),
$$

where $\theta \in(0,1)$ (depending on $s, p)$ and

$$
\lim _{p \searrow 0} \frac{\varphi(s, p)-\varphi(s, 0)}{p}=\partial_{p} \varphi(s, 0)=-\log \lambda_{s}(A),
$$


it follows from the Lebesgue dominated convergence theorem that, for every $t \in$ $(0,1]$,

$$
\left.\frac{d}{d p} \int_{1-t}^{1} \varphi(s, p) d s\right|_{p=+0}=\lim _{p \searrow 0} \int_{1-t}^{1} \frac{\varphi(s, p)-\varphi(s, 0)}{p} d s=-\int_{1-t}^{1} \log \lambda_{s}(A) d s,
$$

where $\left.\frac{d}{d p}(\cdot)\right|_{p=+0}$ means the right derivative at $p=0$. Therefore,

$$
\begin{aligned}
\lim _{p \searrow 0}\left[-\frac{1}{p} \log \left(\frac{1}{t} \int_{1-t}^{1} \lambda_{s}(A)^{-p} d s\right)\right] & =-\left.\frac{d}{d p} \log \left(\frac{1}{t} \int_{1-t}^{1} \lambda_{s}(A)^{-p} d s\right)\right|_{p=+0} \\
& =\frac{1}{t} \int_{1-t}^{1} \log \lambda_{s}(A) d s,
\end{aligned}
$$

which is equivalent to the desired limit formula.

Proof of Theorem 6.11. By Proposition 6.10(a) we may prove that (ii) $\Rightarrow(\mathrm{i})$, so assume that $\int_{0}^{1} \lambda_{s}(B)^{-p} d s<\infty$ for some $p>0$ and $\|A\|_{!} \geq\|B\|_{\text {! for all fully }}$ symmetric derived anti-norms. It suffices to show that, for each $t \in(0,1)$ fixed, if $\left\|A^{-p}\right\|_{(t)}^{-1 / p} \geq\left\|B^{-p}\right\|_{(t)}^{-1 / p}$ for all $p>0$, then $\int_{1-t}^{1} \log \lambda_{s}(A) d s \geq \int_{1-t}^{1} \log \lambda_{s}(B) d s$. Since all the relevant quantities depend on $\lambda(A), \lambda(B)$ restricted on $(1-t, 1)$, we may assume that $A, B \in \mathcal{M}^{+}$, by replacing $A, B$ with $A \wedge \alpha, B \wedge \beta$ where $\alpha:=$ $\lambda_{1-t}(A), \beta:=\lambda_{1-t}(B)$, respectively. Then for every $\delta>0$ we have $\left\|(A+\delta I)^{-p}\right\|_{(t)}^{-1 / p}$ $\geq\left\|A^{-p}\right\|_{(t)}^{-1 / p} \geq\left\|B^{-p}\right\|_{(t)}^{-1 / p}$. Applying Lemma 6.12 to $A+\delta I$ and $B$ yields

$$
\int_{1-t}^{1} \log \left(\lambda_{s}(A)+\delta\right) d s \geq \int_{1-t}^{1} \log \lambda_{s}(B) d s .
$$

Letting $\delta \searrow 0$ gives the desired inequality.

Remark 6.13. Note that there is a $B \in \mathcal{M}^{+}$such that $\int_{0}^{1} \log \lambda_{s}(B) d s>-\infty$ but $\int_{0}^{1} \lambda_{s}(B)^{-p} d s=\infty$ for all $p>0$. For instance, this is the case when $\lambda_{s}(B)=$ $\exp (-1 / \sqrt{1-s})$. For such a $B \in \mathcal{M}^{+}$and every $p>0$, if $\|\cdot\|$ is a fully symmetric norm on $\mathcal{M}^{+}$, then by Proposition 6.4(c) we have

$$
\lim _{\varepsilon \searrow 0}\left\|(B+\varepsilon I)^{-p}\right\| \geq \lim _{\varepsilon \searrow 0}\left\|(B+\varepsilon I)^{-p}\right\|_{1}\|I\|=\int_{0}^{1} \lambda_{s}(B)^{-p} d s\|I\|=\infty .
$$

This means that $\|B\|_{!}=0$ for every fully symmetric derived anti-norm, so (ii) of Theorem 6.11 is satisfied for any $A \in \mathcal{M}^{+}$. Therefore, (ii) $\Rightarrow$ (i) does not hold for general $A, B \in \mathcal{M}^{+}$. This subtle difference between (i) and (ii) does not occur in the matrix case. In the matrix algebra $\mathbb{M}_{n}$, the conditions (i) and (ii) of Theorem 6.11 are equivalent: (i) $\Rightarrow$ (ii) is shown in [7, Lemma 4.10], and (ii) $\Rightarrow$ (i) is implicit in [7, Example 4.5], the discrete version of the anti-norms in (6.2). 
The following is a consequence of Lemma 6.12 .

Corollary 6.14. For every $t \in(0,1]$ the functional

$$
\Delta_{t}(A):=\exp \left(\frac{1}{t} \int_{1-t}^{1} \log \lambda_{s}(A) d s\right), \quad A \in \mathcal{M}^{+},
$$

is a symmetric anti-norm on $\mathcal{M}^{+}$.

Proof. The properties (1)! and (2)! of Definition 2.2 for $\Delta_{t}$ are clear, and (4)! is immediate from the monotone convergence theorem. To show $(3)$ !, we may assume in view of (4)! that $A, B \in \mathcal{M}^{+}$are invertible. Then Lemma 6.12 yields

$$
\Delta_{t}(A)=\lim _{p \searrow 0} t^{-1 / p}\left\|A^{-p}\right\|_{(t)}^{-1 / p}
$$

and the same expressions for $\Delta_{t}(B)$ and $\Delta_{t}(A+B)$. Hence $(3)$ ! for $\Delta_{t}$ follows from the case of the derived anti-norms $\left\|A^{-p}\right\|_{(t)}^{-1 / p}$.

The symmetric anti-norms $\Delta_{t}$ are not derived ones, but (6.3) says that they are on the boundary of the derived anti-norms. In particular, when $t=1$,

$$
\Delta(X):=\exp \left(\int_{0}^{1} \log \lambda_{s}(|X|) d s\right)=\Delta_{1}(|X|), \quad X \in \mathcal{M}
$$

is the Fuglede-Kadison determinant [15]. This is extended to $\overline{\mathcal{M}}$ by Proposition 4.2 and the above expression holds whenever $\int_{0}^{1} \log \lambda_{s}(|X|) d s$ makes sense with $\pm \infty$ permitted. The determinant $\Delta$ has turned out useful in non-commutative $H^{\infty}$ theory (see e.g. $[3,5]$ ).

\section{$\S 7$. Superadditivity with more functions}

For a fully symmetric derived anti-norm, Theorem 5.1 for convex functions can be extended to a considerably larger class of superadditive functions given as follows:

Let $\mathcal{S}$ be the class of functions $\psi:[0, \infty) \rightarrow[0, \infty)$ such that $\psi=f \circ g$ for some superadditive log-concave function $f:[0, \infty) \rightarrow[0, \infty)$ and some superadditive convex function $g:[0, \infty) \rightarrow[0, \infty)$.

Recall that $f:[0, \infty) \rightarrow[0, \infty)$ is log-concave if $f((a+b) / 2) \geq \sqrt{f(a) f(b)}$ for all $a, b \geq 0$, i.e., $\log f:[0, \infty) \rightarrow[-\infty, \infty)$ is concave. A convex function $g:[0, \infty) \rightarrow[0, \infty)$ is superadditive if and only if $g(0)=0$. Note that any function $\psi$ in $\mathcal{S}$ is superadditive and non-decreasing on $[0, \infty)$ with $\psi(0)=0$.

Any superadditive log-concave function $\psi:[0, \infty) \rightarrow[0, \infty)$ is in $\mathcal{S}$. Any convex function $\psi:[0, \infty) \rightarrow[0, \infty)$ with $\psi(0)=0$ is in $\mathcal{S}$. The next examples 
point out some functions in $\mathcal{S}$ which are in the intersection of these two subclasses, or only in one subclass, or in none of them. We implicitly assume that superadditive functions are defined on $[0, \infty)$.

Example 7.1. - The power functions $t \mapsto t^{p}, p \geq 1$, and the angle function at any $\alpha>0, t \mapsto(t-\alpha)_{+}:=\max \{t-\alpha, 0\}$, are superadditive, convex and log-concave. The function $t \mapsto t \arctan t$ is also superadditive, convex and log-concave.

- For any $\gamma>1$, the functions $t \mapsto \sinh t^{\gamma}$ and $t \mapsto t \exp t^{\gamma}$ are superadditive and convex, but not log-concave.

- When $1 \leq \alpha<\beta$, the function $t \mapsto \min \left\{t^{\alpha}, t^{\beta}\right\}$ is superadditive and log-concave, but not convex. The function $t \mapsto t^{\alpha} \exp \left(-1 / t^{\beta}\right)$ has the same properties whenever $\alpha \geq 1$ and $\beta>2 \alpha-1+2 \sqrt{\alpha(\alpha-1)}$. When $0<a<b$, the function $t \mapsto(t-a) \mathbf{1}_{[b, \infty)}(t)$ also has the same properties though it is not continuous.

- For $f(t)=\min \left\{t^{\alpha}, t^{\beta}\right\}$ with $1 \leq \alpha<\beta$ and $g(t)=\sinh t^{\gamma}$ or $t \exp t^{\gamma}$ with $\gamma>1$, $f \circ g$ is a function in $\mathcal{S}$, but neither log-concave nor convex.

Recall that $\mathcal{M}$ stands for a (finite) diffuse algebra. The superadditivity results in this section also hold with $\mathbb{M}_{n}$ in place of $\mathcal{M}$, with similar though simpler proofs. The next theorem is the main result of this section.

Theorem 7.2. Let $A, B \in \overline{\mathcal{M}}^{+}$and let $\psi \in \mathcal{S}$. Then, for any fully symmetric derived anti-norm on $\overline{\mathcal{M}}^{+}$,

$$
\|\psi(A+B)\| ! \geq\|\psi(A)\|_{!}+\|\psi(B)\| ! \cdot
$$

The proof is based on Theorem 3.1 and the next lemma.

Lemma 7.3. Let $\|\cdot\|$ ! be a fully symmetric derived anti-norm on $\mathcal{M}^{+}$. Let $A, B \in \overline{\mathcal{M}}^{+}$. If $f:[0, \infty) \rightarrow[0, \infty)$ is superadditive and log-concave, then

$$
\|f(A+B)\|_{!} \geq\|f(A)\|_{!}+\|f(B)\|_{!} .
$$

Proof. For each $s>0$, since $\lambda(f(A+B)) \geq \lambda(f(A \wedge s+B \wedge s))$, by Proposition 6.10(a) we have $\|f(A+B)\|_{!} \geq\|f(A \wedge s+B \wedge s)\|_{!}$. Since $\|f(A)\|_{!}=$ $\lim _{s \nearrow_{\infty}}\|f(A \wedge s)\|$ ! and similarly for $\|f(B)\|_{\text {! }}$ as in the proof of Theorem 5.1, we may assume that $A, B \in \mathcal{M}^{+}$. We prove that

$$
f(A+B) \prec^{w(\log )} \int_{0}^{1} f\left(\lambda_{t}(A)+\lambda_{t}(B)\right) d F_{t} .
$$

As $f(t)$ is non-decreasing, we have $\lambda(f(A+B)) \geq \lambda(f(A))$ so that $\|f(A+B)\| ! \geq$ $\|f(A)\|$ ! , and similarly $\|f(A+B)\|_{!} \geq\|f(B)\|$ !. Hence, the claimed inequality is obvious if $\|f(A)\|_{!}=0$ or $\|f(B)\|_{!}=0$. So assume that $\|f(A)\| !>0$ and 
$\|f(B)\|_{!}>0$. This implies by Proposition 4.4(b) that $f(A)$ and $f(B)$ are nonsingular, so $f\left(\lambda_{t}(A)\right)>0$ and $f\left(\lambda_{t}(B)\right)>0$ for all $t \in(0,1)$. Hence $f(t)>0$ for all $t>t_{0}:=\min \left\{\lambda_{1}(A), \lambda_{1}(B)\right\}$. Furthermore, thanks to Proposition 6.10(b), it suffices to prove the claimed inequality for $A+\varepsilon I$ and $B+\varepsilon I$ for any $\varepsilon>0$. Thus we can assume that $f(t)>0$ for all $t \geq t_{0}$.

Then, from $\lambda(A+B) \prec \lambda(A)+\lambda(B)$ and the concavity of $\log f$, we have

$$
\log \lambda(f(A+B))=\log f(\lambda(A+B)) \prec^{w} \log f(\lambda(A)+\lambda(B)),
$$

which means that (7.1) holds. As $f$ is further superadditive, (7.1) entails

$$
f(A+B) \prec^{w(\log )} \int_{0}^{1}\left(f\left(\lambda_{t}(A)\right)+f\left(\lambda_{t}(B)\right)\right) d F_{t} .
$$

Therefore, Proposition 6.10(a) implies that

$$
\begin{aligned}
\|f(A+B)\|_{!} & \geq\left\|\int_{0}^{1} f\left(\lambda_{t}(A)\right) d F_{t}\right\|_{!}+\left\|\int_{0}^{1} f\left(\lambda_{t}(B)\right) d F_{t}\right\|_{!} \\
& =\|f(A)\|_{!}+\|f(B)\|_{!} .
\end{aligned}
$$

Proof of Theorem 7.2. As in the proof of the previous lemma, we may and do assume that $A, B \in \mathcal{M}^{+}$. Let $\psi$ in $\mathcal{S}$ be written as $\psi=f \circ g$, with $f$ superadditive $\log$-concave and $g$ superadditive convex. If $\|\psi(A)\|_{!}=0$ or $\|\psi(B)\|_{!}=0$, then the claimed inequality follows as in the proof of the previous lemma. So we assume that $\|\psi(A)\|_{!}>0$ and $\|\psi(B)\|_{!}>0$. This implies that $f\left(\lambda_{t}(g(A))\right)>0$ and $f\left(\lambda_{t}(g(B))\right)>0$ for all $t \in(0,1)$. Hence, $f(t)>0$ for all $t>t_{0}:=$ $\min \left\{\lambda_{1}(g(A)), \lambda_{1}(g(B))\right\}$, so that $f$ is continuous on $\left(t_{0}, \infty\right)$. For every $\varepsilon>0$ let $U, V \in \mathcal{M}$ be unitaries as given in Theorem 3.1. Then

$$
\lambda_{t}(f(g(A+B)+\varepsilon I)) \geq \lambda_{t}\left(f\left(U g(A) U^{*}+V g(B) V^{*}\right)\right)
$$

for all $t \in(0,1)$. By Proposition 6.10(a) and Lemma 7.3, this implies that

$$
\|f(g(A+B)+\varepsilon I)\|_{!} \geq\left\|f\left(U g(A) U^{*}+V g(B) V^{*}\right)\right\|_{!} \geq\|f(g(A))\|_{!}+\|f(g(B))\|_{!} .
$$

Letting $\varepsilon \searrow 0$ yields the claimed inequality thanks to Proposition 6.10(b).

Finally, we return to a general finite von Neumann algebra $\mathcal{N}$ with a faithful normal trace $\tau, \tau(I)=1$, and extend Theorem 7.2 to $\overline{\mathcal{N}}^{+}$with a restriction on derived anti-norms. For this, we start with a fully symmetric norm $\rho$ on the commutative von Neumann algebra $L^{\infty}(0,1)$ with the trace $\int_{0}^{1} \cdot d t$ (expectation). Define a fully symmetric norm $\|\cdot\|_{\rho}$ on $\mathcal{N}$ as

$$
\|X\|_{\rho}:=\rho(\mu(X)), \quad X \in \mathcal{N},
$$


which we call a $\rho$-symmetric norm. This way of constructing symmetric norms is common in the theory of non-commutative Banach function spaces (see e.g. $[11,12])$. Let $\|\cdot\|$ ! be the (fully symmetric) anti-norm on $\mathcal{N}^{+}$that is derived from $\|\cdot\|_{\rho}$ and a $p>0$. For $\mathcal{M}$ diffuse, any fully symmetric norm on $\mathcal{M}$ is $\rho$-symmetric with

$$
\rho(h):=\left\|\int_{0}^{1} h(t) d F_{t}\right\|, \quad h \in L^{\infty}(0,1),
$$

so the restriction here to fully symmetric derived anti-norms is indeed no restriction in the diffuse case. However, on a general $\mathcal{N}$ we have a fully symmetric norm which is not written as a $\rho$-symmetric norm.

Consider the tensor product (diffuse) von Neumann algebra

$$
\mathcal{M}:=\mathcal{N} \bar{\otimes} L^{\infty}(0,1)
$$

with the tensor product trace $\tau \otimes \int_{0}^{1} \cdot d t$, and define the $\rho$-symmetric norm $\|\cdot\|_{\rho}$ on $\mathcal{M}$ and the corresponding derived anti-norm $\|\cdot\|$ ! on $\mathcal{M}^{+}$in the same way as above on $\mathcal{N}$ and $\mathcal{N}^{+}$. Then the following equations are obvious:

$$
\|X\|_{\rho}=\|X \otimes 1\|_{\rho}, \quad X \in \overline{\mathcal{N}} ; \quad\|A\|_{!}=\|A \otimes 1\|_{!}, \quad A \in \overline{\mathcal{N}}^{+} .
$$

Therefore, all the results concerning fully symmetric derived anti-norms on $\mathcal{M}^{+}$ in Section 6 and in this section remain true for the anti-norm $\|\cdot\|$ ! on $\mathcal{N}^{+}$derived from $\|\cdot\|_{\rho}$ as above. In particular, we have

Corollary 7.4. Let $\|\cdot\|$ ! be an anti-norm on $\mathcal{N}^{+}$that is derived from a $\rho$ symmetric norm $\|\cdot\|_{\rho}$ on $\mathcal{N}$ and a $p>0$. Then, for every $A, B \in \overline{\mathcal{N}}^{+}$and every function $\psi$ in $\mathcal{S}$,

$$
\|\psi(A+B)\| ! \geq\|\psi(A)\|_{!}+\|\psi(B)\| ! \cdot
$$

Corollary 7.5. Let $g:[0, \infty) \rightarrow[0, \infty)$ be a convex function with $g(0)=0$, and let $\psi$ be a strictly increasing function in $\mathcal{S}$. Then, for all non-singular $A, B \in \mathcal{N}^{+}$ and all $0<p \leq 1$,

$$
\frac{\tau\left(g^{p}(A+B)\right)}{\tau\left(\psi^{p-1}(A+B)\right)} \geq \frac{\tau\left(g^{p}(A)\right)}{\tau\left(\psi^{p-1}(A)\right)}+\frac{\tau\left(g^{p}(B)\right)}{\tau\left(\psi^{p-1}(B)\right)} .
$$

Proof. By Theorem 5.1 the functional $A \mapsto\left\{\tau\left(g^{p}(A)\right)\right\}^{1 / p}$ is superadditive with finite values on $\mathcal{N}^{+}$. By the previous corollary, $A \mapsto\left\{\tau\left(\psi^{p-1}(A)\right)\right\}^{1 /(p-1)}$ is superadditive with strictly positive finite values on the non-singular part of $\mathcal{N}^{+}$. Hence, their $p$-weighted geometric mean is again superadditive on the non-singular part of $\mathcal{N}^{+}$. 
Note that $\|\cdot\|_{(t)}$ is a $\rho$-symmetric norm and (6.3) is valid in $\mathcal{N}^{+}$. Hence, through Corollary 7.4 applied to the anti-norm derived from $\|\cdot\|_{(t)}$, we have

$$
\Delta_{t}(\psi(A+B)) \geq \Delta_{t}(\psi(A))+\Delta_{t}(\psi(B)), \quad A, B \in \overline{\mathcal{N}}^{+},
$$

for all $t \in(0,1]$ and all $\psi$ in $\mathcal{S}$. For the Fuglede-Kadison determinant $\Delta$, since $\Delta(\sqrt{\psi \omega}(A))=\{\Delta(\psi(A)) \Delta(\omega(A))\}^{1 / 2}$ for $\psi, \omega:[0, \infty) \rightarrow[0, \infty)$ and $A \in \overline{\mathcal{N}}^{+}$, we furthermore have

Corollary 7.6. Let $\psi, \omega \in \mathcal{S}$. Then, for all $A, B \in \overline{\mathcal{N}}^{+}$,

$$
\Delta(\sqrt{\psi \omega}(A+B)) \geq \Delta(\sqrt{\psi \omega}(A))+\Delta(\sqrt{\psi \omega}(B)) .
$$

This is a substantial generalization of the Minkowski inequality for $\Delta(A)$ on $\mathcal{M}^{+}$given in [3] as a consequence of a variational expression of $\Delta$. Note that the concavity of $A \mapsto \Delta(f(A))$ on $\mathcal{M}^{\text {sa }}$ for a positive concave function $f$ was shown in [22] (a similar result for matrices is in [6]).

\section{Acknowledgements}

Research of J.-C. Bourin was supported by ANR 2011-BS01-008-01. Research of F. Hiai was supported by Grant-in-Aid for Scientific Research (C)26400103.

\section{References}

[1] C. A. Akemann, J. Anderson and G. K. Pedersen, Triangle inequalities in operator algebras, Linear Multilinear Algebra 11 (1982), 167-178. Zbl 0485.46029 MR 0650729

[2] J. S. Aujla and J.-C. Bourin, Eigenvalue inequalities for convex and log-convex functions, Linear Algebra Appl. 424 (2007), 25-35. Zbl 1132.15015 MR 2324372

[3] W. B. Arveson, Analyticity in operator algebras, Amer. J. Math. 89 (1967), 578-642. Zbl 0183.42501 MR 0223899

[4] R. Bhatia, Matrix analysis, Springer, New York, 1997. Zbl 0863.15001 MR 1477662

[5] D. P. Blecher and L. E. Labuschagne, Von Neumann algebraic $H^{\infty}$ theory, in Function spaces, Contemp. Math. 435, Amer. Math. Soc., 2007, 89-114. Zbl 1145.46040 MR 2359421

[6] J.-C. Bourin and F. Hiai, Norm and anti-norm inequalities for positive semi-definite matrices, Int. J. Math. 22 (2011), 1121-1138. Zbl 1239.15014 MR 2826557

[7] _ Jensen and Minkowski inequalities for operator means and anti-norms, Linear Algebra Appl. 456 (2014), 22-53. Zbl 1293.15017 MR 3223888

[8] J.-C. Bourin and E.-Y. Lee, Unitary orbits of Hermitian operators with convex or concave functions, Bull. London Math. Soc. 44 (2012), 1085-1102. Zbl 1255.15028 MR 3007642

[9] L. G. Brown and H. Kosaki, Jensen's inequality in semi-finite von Neumann algebras, J. Operator Theory 23 (1990), 3-19. Zbl 0718.46026 MR 1054812

[10] K. M. Chong, Doubly stochastic operators and rearrangement theorems, J. Math. Anal. Appl. 56 (1976), 309-316. Zbl 0364.60112 MR 0417844 
[11] P. G. Dodds, T. K.-Y. Dodds and B. de Pagter, Non-commutative Banach function spaces, Math. Z. 201 (1989), 583-597. Zbl 0653.46061 MR 1004176

[12] Noncommutative Köthe duality, Trans. Amer. Math. Soc. 339 (1993), 717-750. Zbl 0801.46074 MR 1113694

[13] T. Fack and H. Kosaki, Generalized s-numbers of $\tau$-measurable operators, Pacific J. Math. 123 (1986), 269-300. Zbl 0617.46063 MR 0840845

[14] J. Fang, D. Hadwin, E. Nordgren and J. Shen, Tracial gauge norms on finite von Neumann algebras satisfying the weak Dixmier property, J. Funct. Anal. 255 (2008), 142-183. Zbl 1156.46040 MR 2417813

[15] B. Fuglede and R. Kadison, Determinant theory in finite factors, Ann. of Math. 55 (1952), 520-530. Zbl 0046.33604 MR 0052696

[16] F. Hiai, Spectral relations and unitary mixing in semifinite von Neumann algebras, Hokkaido Math. J. 17 (1988), 117-137. Zbl 0641.46042 MR 0928470

[17] F. Hiai and Y. Nakamura, Majorizations for generalized s-numbers in semifinite von Neumann algebras, Math. Z. 195 (1987), 17-27. Zbl 0598.46039 MR 0888123

[18] _ Distance between unitary orbits in von Neumann algebras, Pacific J. Math. 138 (1989), 259-294. Zbl 0667.46044 MR 0996202

[19] Closed convex hulls of unitary orbits in von Neumann algebras, Trans. Amer. Math. Soc. 323 (1991), 1-38. Zbl 0743.46057 MR 0984856

[20] C. J. Himmelberg, Measurable relations, Fund. Math. 87 (1975), 53-72. Z Zbl 0296.28003 MR 0367142

[21] H. Kosaki, On the continuity of the map $\varphi \rightarrow|\varphi|$ from the predual of a $W^{*}$-algebra, J. Funct. Anal. 59 (1984), 123-131. Zbl 0584.46050 MR 0763779

[22] E. H. Lieb and G. K. Pedersen, Convex multivariable trace functions, Rev. Math. Phys. 14 (2002), 631-648. Zbl 1034.46050 MR 1932659

[23] S. Lord, F. Sukochev and D. Zanin, Singular traces: theory and applications, de Gruyter Stud. Math. Phys. 46, de Gruyter, Berlin, 2013. Zbl 1275.47002 MR 309977

[24] M. Marcus and L. Lopes, Inequalities for symmetric functions and Hermitian matrices, Canad. J. Math. 9 (1957), 305-312. Z Zbl 0079.02103 MR 0084541

[25] A. W. Marshall, I. Olkin and B. C. Arnold, Inequalities: theory of majorization and its applications, 2nd ed., Springer, New York, 2011. Zbl 1219.26003 MR 2759813

[26] D. Petz, Spectral scale of self-adjoint operators and trace inequalities, J. Math. Anal. Appl. 109 (1985), 74-82. Zbl 0655.47032 MR 0796042

[27] M. Takesaki, Theory of operator algebras. I, Springer, New York, 1979. Z Zbl 0436.46043 MR 1873025

[28] K. Watanabe, Some results on non-commutative Banach function spaces, Math. Z. 210 (1992), 555-572. Zbl 0759.46057 MR 1175721 